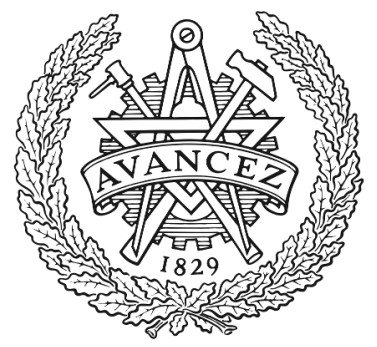

CHALMERS

UNIVERSITY OF TECHNOLOGY

\title{
Convergence Acceleration of the Harmonic Balance Method using a Time-Level Preconditioner
}

Downloaded from: https://research.chalmers.se, 2023-04-26 09:27 UTC

Citation for the original published paper (version of record):

Lindblad, D., Andersson, N. (2020). Convergence Acceleration of the Harmonic Balance Method using a Time-Level Preconditioner. AIAA Journal, 58(11): 4908-4922.

http://dx.doi.org/10.2514/1.J059276

N.B. When citing this work, cite the original published paper. 


\title{
Convergence Acceleration of the Harmonic Balance Method using a Time-Level Preconditioner
}

\author{
Daniel Lindblad*, and Niklas Andersson ${ }^{\dagger}$ \\ Chalmers University of Technology, Gothenburg, SE-412 96, Sweden
}

\begin{abstract}
The Harmonic Balance method is nowadays widely applied for numerically solving problems that are known to possess time-periodic solutions. Key reasons for its success are its wide range of applicability, relative ease of implementation, and computational efficiency compared to timeaccurate approaches. The computational efficiency of the Harmonic Balance method is partly derived from the fact that it searches directly for a periodic solution, instead of integrating the governing equations in time until a periodic solution is reached. Convergence acceleration techniques such as multigrid, implicit residual smoothing and local time stepping may also be used to improve the efficiency of the Harmonic Balance method. This paper considers another option for accelerating convergence, namely a novel time-level preconditioner that can be applied to the Harmonic Balance residual locally in each computational cell. The benefit of employing this preconditioner is analyzed both from a theoretical perspective, by performing a rigorous stability analysis based on the linearized Euler equations, and from a numerical perspective, by applying it to two test cases.
\end{abstract}

\section{Nomenclature}

Roman/Greek

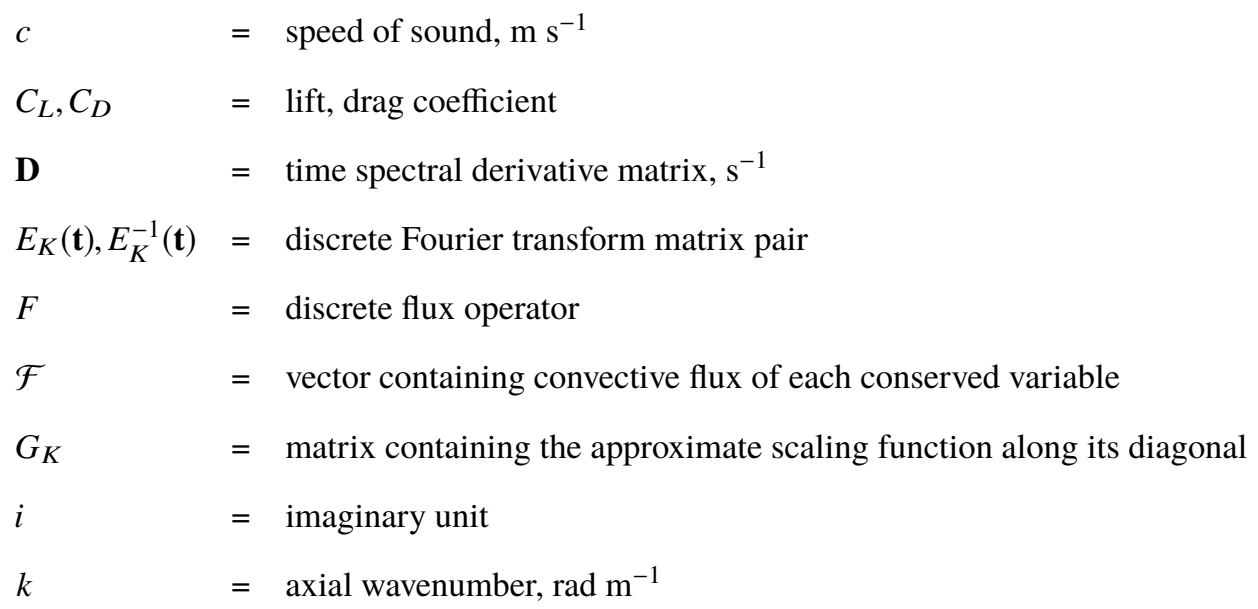

*Ph.D. Student, Department of Mechanics and Maritime Sciences, Hörsalsvägen 7A, SE-412 96 Gothenburg.

${ }^{\dagger}$ Associate Professor, Department of Mechanics and Maritime Sciences, Hörsalsvägen 7A, SE-412 96 Gothenburg. 


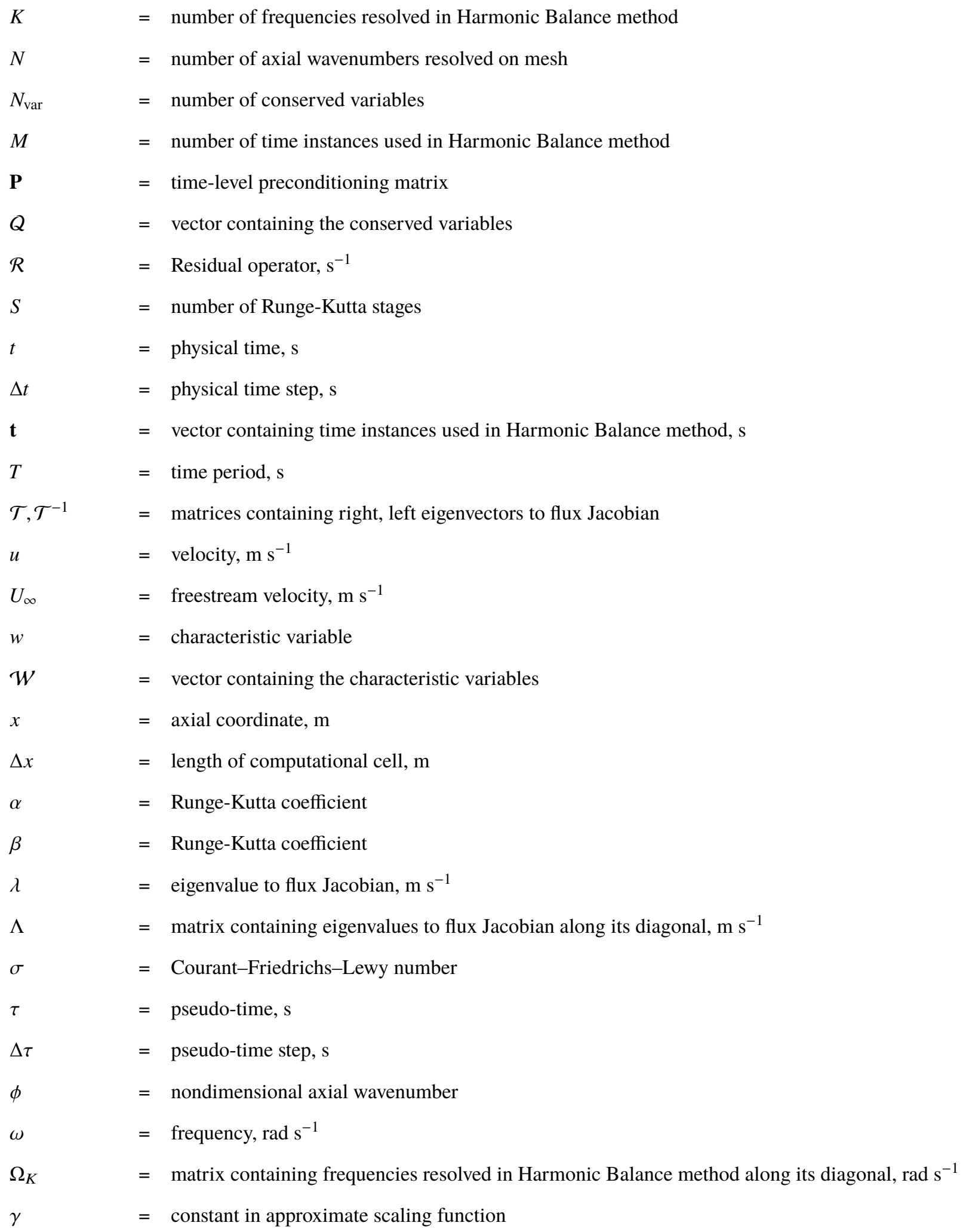




$$
\begin{aligned}
& \square_{e}=\text { east face of cell } \\
& \square_{j} \quad=\text { cell index } \\
& \square_{k}=\text { frequency index } \\
& \square_{l}=\text { physical time index } \\
& \square_{m}=\text { pseudo-time index } \\
& \square_{n} \quad=\text { spatial wavenumber index } \\
& \square_{p} \quad=\text { characteristic variable index } \\
& \square_{w}=\text { west face of cell } \\
& \bar{\square} \quad=\text { spatial+temporal average } \\
& \hat{\square} \quad=\text { temporal Fourier transform } \\
& \hat{\Delta} \quad=\text { spatial + temporal Fourier transform }
\end{aligned}
$$

\section{Introduction}

T UmERICAL simulations are today important tools in several engineering fields. In particular, the field of machines. A characteristic feature of turbomachines is that fluid flows inside them are inherently unsteady and time-periodic. This feature has promoted a lot of research within the turbomachinery community on numerical methods that are tailored for solving time periodic problems, both on the fluid and the structural side [1, 2].

Early research on time-periodic flows inside turbomachines focused on solving a linearized set of equations. The advantage of this approach is that it decouples each frequency in the solution and that it often is a good model of the flow. One of the first approaches that incorporated nonlinear terms into the governing equations was the Nonlinear Harmonic method [3]. Later, the Harmonic Balance (HB) method was introduced in the Computational Fluid Dynamics (CFD) community by Hall et al. [4]. Hall et al. formulated their HB method in the time domain. An equivalent formulation in the frequency domain is the Nonlinear Frequency Domain (NLFD) method developed by McMullen [5]. In both these methods, the solution is assumed to take on the form of a truncated Fourier series in time, with spatially varying coefficients. This allows the time derivative to be calculated exactly and then be included in the governing equations as a source term. This effectively transforms the problem from finding the time evolution of the governing equations, into a steady state problem for finding the Fourier coefficients. An appealing feature of the HB and NLFD methods is that they can be readily incorporated into an existing CFD code. In addition to this, the HB/NLFD methods can account for nonlinear interaction among the resolved frequencies. Since their introduction into the CFD community, these methods have therefore been adopted by many researchers and have proven to be both accurate and computationally efficient [6. 10$]$. 
Early applications of the HB method showed that it can give rise to numerical instabilities when a large number of frequencies is included in the simulation and the equations are solved using explicit pseudo-time integration [4]. McMullen et al. [11] analyzed the stability of the NLFD method and showed that the time derivative source term adds an imaginary part to the eigenvalue spectrum of the spatial discretization. They further concluded that this will lead to stability issues if the time derivative source term is large enough to push the eigenvalue spectrum outside the limited stability region of an explicit pseudo-time integration scheme. These findings are indeed consistent with the stability issues reported by Hall et al. [4]. McMullen et al. [11] initially proposed to overcome the stability issue associated with the time derivative source term by treating it in a point implicit manner inside an explicit Runge-Kutta scheme. Later, it was shown by the same authors that this approach rotates the stability region of the Runge-Kutta scheme in the complex plane such that it no longer covers parts of the imaginary axis [5, 12]. From this, it was concluded that the point implicit treatment of the time derivative source term was unsuitable, at least when a central discretization of convection terms is used since the eigenvalue spectrum then is purely imaginary. Other authors have however reported that the stability of the HB method can be improved with a point implicit treatment of the time derivative source term [9]. This suggests that the stability of the point implicit treatment depends on which pseudo-time integration and spatial discretization scheme it is applied to.

Instead of applying the point implicit treatment, McMullen [5] scales the pseudo-time step used in his NLFD solver so that each frequency component of the solution is updated with the largest possible pseudo-time step allowed by his explicit Runge-Kutta solver. Later, van der Weide et al. [13] derived a similar restriction on the pseudo-time step. They also noted that it is necessary to perform a matrix-vector multiplication in each cell and iteration when this restriction is implemented in the time domain HB method. In order to avoid this overhead, van der Weide et al. [13] proposed to use the smallest pseudo-time step, corresponding to the highest frequency, when the time domain HB method is employed. Similar pseudo-time step restrictions have since then been adopted by several other authors in their respective time domain HB implementations [10, 14, 15]. An extensive stability analysis of the HB method has also been performed by Gentilli [16], who showed that the stability of an explicit HB solver depends both on the classical CFL number, as well as an additional parameter referred to as the grid reduced frequency. In his work, Gentillialso derives exact pseudo-time step restrictions for a four stage Runge-Kutta solver and a set of spatial discretization schemes. These restrictions can in fact be seen as tailored versions of the pseudo-time step restriction developed earlier by van der Weide et al. [13].

The restrictions on the pseudo-time step associated with explicit schemes can in principle be removed by employing an A-stable pseudo-time integration scheme, e.g. backward Euler, to integrate the equations. When this approach is followed for the HB method, the Jacobian matrix will be $M$ times larger than for a steady state computation, where $M$ denotes the number of time instances used in the HB method [17, 18]. This Jacobian will further be structured into $M \times M$ blocks, in which the diagonal sub blocks contain the same flux Jacobian that is used in a steady state problem, and the off-diagonal sub blocks contain the linearization of the time derivative source term. This structure reduces the 
diagonal dominance of the linear system when the time derivative source term is large [14], and was found to impact the convergence rate of the linear solver in [18]. It is interesting to note that the ratio derived in [14] to quantify the diagonal dominance of the linear system is equivalent to the grid reduced frequency introduced by Gentilli [16] to quantify the stability of explicit HB solvers. One way to strengthen the diagonal dominance of the linear system is to restrict the pseudo-time step used in the backward Euler method. Su and Yuan [14] do this based on the formula originally proposed by van der Weide et al. [13]. Another approach, adopted by Antheaume and Corre [19], is to add the norm of the time derivative source term to the diagonal of the linear system. In either of these cases, the linear system is better conditioned at the cost of slower convergence in pseudo-time.

If the time derivative source term on the other hand is treated explicitly in the backward Euler scheme, the resulting linear system decouples across all time instances. This approach facilitates the implementation of the HB method into an existing CFD code but has unfortunately been proven to be unconditionally unstable [20]. Woodgate and Badcock [18] and Sicot et al. [21] have also verified this stability issue numerically. Several schemes have therefore been proposed to circumvent the problem of having to solve a large linear system, while at the same time retaining numerical stability. Sicot et al. [21] use a block-Jacobi symmetric successive over-relaxation (BJ-SSOR) technique to solve the linear system. Another approach is the two step approximate factorization method developed by Thomas et al. [17]. Implicit residual smoothing using the LU-SGS method combined with the block Jacobi method to treat the time spectral derivative source term implicitly has also been shown to greatly improve the stability of an explicit Runge-Kutta solver in [22].

In summary, a wealth of research has been conducted on numerical methods for solving the Harmonic Balance form of the Navier-Stokes equations in fluid dynamics. Most of these methods are based on employing either explicit or implicit pseudo-time integration to converge the discrete residual to zero. In both these cases, it has been shown that the time derivative source term introduces new challenges. For an explicit scheme, the stability of the pseudo-time integration may be compromised, whereas, for an implicit scheme, the linear system becomes harder to solve. This paper starts by deriving the eigenvalue spectra obtained from using the HB method and finite volume method to respectively discretize the Euler equations in time and space. Based on these results, three different methods available in the literature that potentially could be used to stabilize explicit pseudo-time integration of the discrete equations are analyzed. These include the point implicit Runge-Kutta method proposed by McMullen et al. [11] and Campobasso and Baba-Ahmadi [9], the HB source term stabilization matrix used by Thomas et al. [17] in their two step approximate factorization approach, and the pseudo-time step restriction proposed by van der Weide et al. [13]. Based on the knowledge gained from this analysis, a novel time-level preconditioner is then derived for the time domain HB method. This preconditioner scales the pseudo-time step with which each frequency component is being updated, without having to explicitly convert the time domain HB solution into the frequency domain. The benefit of this time-level preconditioner is also demonstrated for two test cases. In the first case, a spatially uniform flow inside a domain which oscillates in the axial direction is simulated, and in the second case, laminar vortex shedding behind a cylinder is simulated. 


\section{Method}

\section{A. Harmonic Balance Temporal Discretization}

The Harmonic Balance method proposed by Hall et al. [4] can be used to compute time-periodic solutions to a wide variety of differential equations. In this work, the HB method will be applied to governing equations in fluid dynamics. For the stability analysis presented later, the one dimensional Euler equations for a perfect, ideal gas will be considered. These equations may be written in compact form as

$$
\frac{\partial Q}{\partial t}+\frac{\partial \mathcal{F}(Q)}{\partial x}=0
$$

In this equation, $Q \in \mathbb{R}^{N_{\text {var }}}$ is a vector that contains the conserved variables and $\mathcal{F}: \mathbb{R}^{N_{\text {var }}} \rightarrow \mathbb{R}^{N_{\text {var }}}$ a function that computes the convective flux of each conserved variable. The convective flux is a nonlinear function of the conserved variables. This implies that periodic solutions to Eq. (1) can contain an infinite number of sinusoids with different frequencies. Computing the amplitude of all these sinusoids is of course not possible from a computational point of view. Therefore, the HB method considers approximate solutions to Eq. (1) instead

$$
Q(t)=\sum_{k=-K}^{K} \hat{Q}_{k} e^{i \omega_{k} t}
$$

Here, $\omega_{k}=k \omega$ is the frequency of the $k^{\text {th }}$ harmonic and $\omega$ the fundamental frequency of the problem.

The first step in deriving the HB form of Eq. (1) is to substitute Eq. (2) into Eq. (1). This yields the following equation

$$
\mathbf{E}_{K}^{-1}(t) \boldsymbol{\Omega}_{K} \hat{Q}_{K}+\frac{\partial \mathcal{F}\left(\mathbf{E}_{K}^{-1}(t) \hat{Q}_{K}\right)}{\partial x}=0
$$

Here, $\mathbf{E}_{K}^{-1}(t)=E_{K}^{-1}(t) \otimes \mathbf{I}$ and $\boldsymbol{\Omega}_{K}=\Omega_{K} \otimes \mathbf{I}$, where $\otimes$ denotes the Kronecker product, $\mathbf{I}$ is a diagonal matrix of size $N_{\text {var }} \times N_{\text {var }}$, and

$$
E_{K}^{-1}(t)=\left[1 e^{i \omega_{1} t} e^{i \omega_{2} t} \cdots e^{i \omega_{-1} t}\right] \quad \Omega_{K}=\left[\begin{array}{cccccc}
0 & 0 & \cdots & & & 0 \\
0 & i \omega_{1} & 0 & \cdots & & 0 \\
\vdots & 0 & i \omega_{2} & 0 & \cdots & 0 \\
& \vdots & 0 & \ddots & & 0 \\
& & \vdots & & & 0 \\
0 & 0 & 0 & 0 & 0 & i \omega_{-1}
\end{array}\right]
$$


The vector $\hat{Q}_{K} \in \mathbb{C}^{(2 K+1) \times N_{\text {var }}}$ in Eq. (3) is further structured into $2 K+1$ sub-vectors, in which the $k^{\text {th }}$ sub-vector contains $\hat{Q}_{k}$ in Eq. (2).

The derivation of the HB form of Eq. (1) continues by imposing the requirement that Eq. (3) should be satisfied at $M=2 K+1$ time instances that are uniformly distributed over the time period $T=2 \pi / \omega$ [4]. This requirement may be stated as

$$
\mathbf{E}_{K}^{-1}(\mathbf{t}) \boldsymbol{\Omega}_{K} \hat{Q}_{K}+\frac{\partial \mathcal{F}^{*}\left(\mathbf{E}_{K}^{-1}(\mathbf{t}) \hat{Q}_{K}\right)}{\partial x}=0
$$

Here,

$$
\mathbf{t}=\left[t_{0}, t_{1}, \ldots, t_{M-1}\right]^{T}
$$

is a vector that contains the time instances $t_{l}=l T / M$ at which Eq. (3) is required to be satisfied, $\mathbf{E}_{K}^{-1}(\mathbf{t})=E_{K}^{-1}(\mathbf{t}) \otimes \mathbf{I}$, and

$$
E_{K}^{-1}(\mathbf{t})=\left[\begin{array}{ccccc}
1 & e^{i \omega_{1} t_{0}} & e^{i \omega_{2} t_{0}} & \cdots & e^{i \omega_{-1} t_{0}} \\
1 & e^{i \omega_{1} t_{1}} & e^{i \omega_{2} t_{1}} & \cdots & e^{i \omega_{-1} t_{1}} \\
\vdots & \vdots & \vdots & & \vdots \\
1 & e^{i \omega_{1} t_{M-1}} & e^{i \omega_{2} t_{M-1}} & \cdots & e^{i \omega_{-1} t_{M-1}}
\end{array}\right]
$$

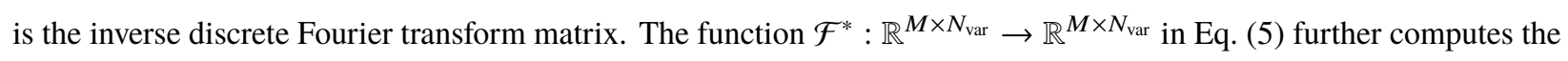
convective flux of each conserved variable at each time instant. The argument to this function is the realization of the Fourier coefficients in $\hat{Q}_{K}$ at the time instances in $\mathbf{t}$

$$
Q^{*}=\mathbf{E}_{K}^{-1}(\mathbf{t}) \hat{Q}_{K}
$$

The inverse discrete Fourier transform matrix defined in Eq. (7) is always invertible for the given choice of frequencies and time instances. From this, it follows that $\mathbf{E}_{K}^{-1}(\mathbf{t})=E_{K}^{-1}(\mathbf{t}) \otimes \mathbf{I}$ is invertible as well. The inverse to this matrix is given by $\mathbf{E}_{K}(\mathbf{t})=E_{K}(\mathbf{t}) \otimes \mathbf{I}$, where

$$
E_{K}(\mathbf{t})=\frac{1}{M}\left[\begin{array}{ccccc}
1 & 1 & 1 & \cdots & 1 \\
e^{-i \omega_{1} t_{0}} & e^{-i \omega_{1} t_{1}} & e^{-i \omega_{1} t_{2}} & \cdots & e^{-i \omega_{1} t_{M-1}} \\
\vdots & \vdots & \vdots & & \vdots \\
e^{-i \omega_{-1} t_{0}} & e^{-i \omega_{-1} t_{1}} & e^{-i \omega_{-1} t_{2}} & \cdots & e^{-i \omega_{-1} t_{M-1}}
\end{array}\right]
$$


is the standard discrete Fourier transform matrix. It should be noted that the uniform time sampling employed here is only appropriate for problems that contain a single fundamental frequency and its harmonics. For multi frequency problems, it is better to use a (possibly non-uniform) time sampling that minimizes the condition number of the inverse discrete Fourier transform matrix, since this ensures that $E_{K}^{-1}(\mathbf{t})$ is invertible, and also can help minimize aliasing [15, 23, -25].

The matrix $\mathbf{E}_{K}(\mathbf{t})$ may be used to compute the Fourier coefficients of the conserved variables from the vector $Q^{*}$ as follows

$$
\hat{Q}_{K}=\mathbf{E}_{K}(\mathbf{t}) Q^{*}
$$

This relation together with Eq. (8) may now be inserted in Eq. (5) to obtain the time domain formulation of the HB method

$$
\underbrace{\mathbf{E}_{K}^{-1}(\mathbf{t}) \boldsymbol{\Omega}_{K} \mathbf{E}_{K}(\mathbf{t})}_{\mathbf{D}} Q^{*}+\frac{\partial \mathcal{F}^{*}\left(Q^{*}\right)}{\partial x}=0
$$

The matrix $\mathbf{D}$ introduced in the above equation is often referred to as the time spectral derivative matrix [4]. It represents a finite difference operator of spectral order, that returns the exact time derivative of $Q$ at the time instances in $\mathbf{t}$ as long as the solution contains at most $K$ harmonics.

The HB method may also be formulated in the frequency domain, in which case it is often referred to as the Nonlinear Frequency Domain (NLFD) method [12]. The NLFD formulation of Eq. (1) is obtained by multiplying Eq. (5) by $\mathbf{E}_{K}(\mathbf{t})$. This yields the following result

$$
\mathbf{\Omega}_{K} \hat{\mathbf{Q}}_{K}+\mathbf{E}_{K}(\mathbf{t}) \frac{\partial \mathcal{F}^{*}\left(\mathbf{E}_{K}^{-1}(\mathbf{t}) \hat{\mathbf{Q}}_{K}\right)}{\partial x}=0
$$

The frequency domain formulation of the HB method presented in the above equation will be used for the upcoming stability analysis. The results presented will however be equally valid for the time domain HB method. This follows from the fact that Eqs 11 and 12 are equivalent.

\section{B. Finite Volume Spatial Discretization}

Before the stability analysis is performed in the next section, Eq. 12 must also be discretized in space, for which the finite volume method is used. The first step in this process is to rewrite Eq. (12) as follows

$$
\mathbf{\Omega}_{K} \hat{\boldsymbol{Q}}_{K}+\mathbf{E}_{K}(\mathbf{t}) \frac{\partial \mathcal{F}^{*}\left(Q^{*}\right)}{\partial Q^{*}} \frac{\partial Q^{*}}{\partial x}=0
$$


Here, $\frac{\partial \mathcal{F}^{*}\left(Q^{*}\right)}{\partial Q^{*}}$ denotes the flux Jacobian. It is a block diagonal matrix that contains $M$ diagonal sub-blocks. The $l^{\text {th }}$ sub-block further contains the steady state flux Jacobian $\left(\frac{\partial \mathcal{F}(Q)}{\partial Q}\right)$, evaluated from the solution at $t_{l}$. If all flow perturbations are small in amplitude, however, the flux Jacobian may be approximated by evaluating it from a spatially+temporally averaged mean state. This yields the following linearized form of Eq. (13)

$$
\mathbf{\Omega}_{K} \hat{\boldsymbol{Q}}_{K}+\mathbf{E}_{K}(\mathbf{t}) \frac{\partial \mathcal{F}^{*}(\overline{\mathbf{Q}})}{\partial Q^{*}} \frac{\partial Q^{*}}{\partial x}=0
$$

Since the elements in the flux Jacobian have been assumed to be the same for all time instances, it commutes with $\mathbf{E}_{K}(\mathbf{t})$ [16]. Equation (14) may therefore be rewritten as

$$
\mathbf{\Omega}_{K} \hat{\boldsymbol{Q}}_{K}+\frac{\partial \mathcal{F}^{*}(\overline{\boldsymbol{Q}})}{\partial \mathbf{Q}^{*}} \frac{\partial \hat{\boldsymbol{Q}}_{K}}{\partial x}=0
$$

The next step in computing the spatial discretization of Eq. 12 is to introduce the diagonalization of the flux Jacobian

$$
\frac{\partial \mathcal{F}^{*}(\overline{\boldsymbol{Q}})}{\partial \mathcal{Q}^{*}}=\mathcal{T}^{*} \Lambda^{*} \mathcal{T}^{*-1}
$$

In this equation, $\mathcal{T}^{*}, \Lambda^{*}$ and $\mathcal{T}^{*-1}$ are all block diagonal matrices that respectively contain the right eigenvectors, eigenvalues and left eigenvectors of $\frac{\partial \mathcal{F}^{*}(\bar{Q})}{\partial Q^{*}}$. For the one dimensional Euler equations, the eigenvalues are given by $\bar{u}$, $\bar{u}-\bar{c}$ and $\bar{u}+\bar{c}$, where $\bar{u}$ and $\bar{c}$ respectively denote the mean axial velocity and mean speed of sound. By substituting Eq. (16) into Eq. [15] and use the fact that the matrices $\mathcal{T}^{*}$ and $\mathcal{T}^{*-1}$ are constant in time and space, the following relation may be derived

$$
\mathbf{\Omega}_{K} \hat{\mathcal{W}}_{K}+\Lambda^{*} \frac{\partial \hat{\mathcal{W}}_{K}}{\partial x}=0
$$

In this equation, the Fourier coefficients of the characteristic variables were introduced. They are defined as

$$
\hat{\mathcal{W}}_{K}=\mathcal{T}^{*-1} \hat{Q}_{K}
$$

Equation (17) represents a set of uncoupled transport equations for the Fourier coefficients of the characteristic variables. The fact that these equations are uncoupled greatly facilitates the stability analysis, since it allows the propagation of a single Fourier coefficient of a single characteristic variable to be considered at a time. Let the $k^{\text {th }}$ Fourier coefficient of the $p^{\text {th }}$ characteristic variable be denoted $\hat{w}_{k}^{p}$. From Eq. (17), it follows that the transport equation for this variable may be written as

$$
i \omega_{k} \hat{w}_{k}^{p}+\lambda_{p} \frac{\partial \hat{w}_{k}^{p}}{\partial x}=0
$$




\begin{tabular}{cccccc}
\hline Abbreviation & Order of Accuracy & $a_{1}$ & $a_{2}$ & $a_{3}$ & $a_{4}$ \\
FOU & $O(\Delta x)$ & 0 & 1 & 0 & 0 \\
TOU & $O\left(\Delta x^{3}\right)$ & $-1 / 6$ & $5 / 6$ & $2 / 6$ & 0 \\
TOU-LD & $O\left(\Delta x^{3}\right)$ & $-9 / 96$ & $59 / 96$ & $53 / 56$ & $-7 / 96$ \\
\hline
\end{tabular}

Table 1 Summary of numerical schemes.

Here, $\lambda_{p}$ denotes the eigenvalue corresponding to the $p^{\text {th }}$ characteristic variable. As can be seen from the above relation, this eigenvalue represents the speed with which the corresponding characteristic variable propagates.

The spatial discretization is performed on a uniform grid with $2 N+1$ equally sized cells of size $\Delta x$. By integrating Eq. (19) over one cell in this grid and then apply Gauss' divergence theorem to the convection term, one obtains

$$
i \omega_{k} \Delta x \hat{w}_{k, j}^{p}+\lambda_{p}\left(\hat{w}_{k, e}^{p}-\hat{w}_{k, w}^{p}\right)=0
$$

In this equation, $\hat{w}_{k, j}^{p}, \hat{w}_{k, w}^{p}$ and $\hat{w}_{k, e}^{p}$ respectively denote the average value, west face value and east face value of the $j^{\text {th }}$ cell. The face values must be estimated using some appropriate flux scheme. In the G3D::Flow code used by the present authors, they are calculated as follows [26]

$$
\begin{array}{lllll}
\hat{w}_{k, e}^{p}=a_{1} \hat{w}_{k, j-1}^{p} & +a_{2} \hat{w}_{k, j}^{p} & +a_{3} \hat{w}_{k, j+1}^{p} & +a_{4} \hat{w}_{k, j+2}^{p}, & \lambda_{p}>0 \\
\hat{w}_{k, w}^{p}=a_{1} \hat{w}_{k, j-2}^{p} & +a_{2} \hat{w}_{k, j-1}^{p} & +a_{3} \hat{w}_{k, j}^{p} & +a_{4} \hat{w}_{k, j+1}^{p}, & \lambda_{p}>0 \\
\hat{w}_{k, e}^{p}=a_{1} \hat{w}_{k, j+2}^{p} & +a_{2} \hat{w}_{k, j+1}^{p} & +a_{3} \hat{w}_{k, j}^{p} & +a_{4} \hat{w}_{k, j-1}^{p}, & \lambda_{p}<0 \\
\hat{w}_{k, w}^{p}=a_{1} \hat{w}_{k, j+1}^{p} & +a_{2} \hat{w}_{k, j}^{p} & +a_{3} \hat{w}_{k, j-1}^{p} & +a_{4} \hat{w}_{k, j-2}^{p}, & \lambda_{p}<0
\end{array}
$$

The coefficients $a_{1}-a_{4}$ in the above equation define the flux scheme. In the present work, three different upwind schemes $\left(a_{1}+a_{2}>a_{3}+a_{4}\right)$ have been considered. More details regarding these schemes, including their order of accuracy, are provided in Table 1 .

By applying Eq. 21] to Eq. 20, the following is obtained

$$
i \omega_{k} \hat{w}_{k, j}^{p}+\frac{\lambda_{p}}{\Delta x} F\left(\hat{w}_{k, j-2}^{p}, \ldots, \hat{w}_{k, j+2}^{p}\right)=0
$$

Here, $F$ is a linear operator that simply computes a weighed sum of the average cell values surrounding the $j^{\text {th }}$ cell. The above equation represents the spatially and temporally discretized one dimensional Euler equations in the frequency+characteristic variable domain.

As noted in the introduction, many codes today use local time stepping to converge the spatially+temporally discretized residual to zero. This implies that the following set of equations are integrated until a steady state is reached 


$$
\frac{\partial \hat{w}_{k, j}^{p}}{\partial \tau}+i \omega_{k} \hat{w}_{k, j}^{p}+\frac{\lambda_{p}}{\Delta x} F\left(\hat{w}_{k, j-2}^{p}, \ldots, \hat{w}_{k, j+2}^{p}\right)=0
$$

In the next section, the stability of this pseudo-time integration will be analyzed when it is performed with explicit Runge-Kutta schemes.

\section{Stability Analysis}

The first step in the stability analysis is to introduce eigenvectors to the spatial operator in Eq. (23). In this work, it is assumed that the solution is periodic in space. Under this assumption, the following spatial Fourier mode represents an eigenvector to $F[27$

$$
\begin{aligned}
\hat{w}_{k, j}^{p} & =\hat{\hat{w}}_{k, n}^{p} e^{i k_{n} x_{j}} \\
& =\hat{\hat{w}}_{k, n}^{p} e^{i j \phi}
\end{aligned}
$$

Here, $\hat{\hat{w}}_{k, n}^{p}$ is the spatio-temporal Fourier coefficient for the $n^{\text {th }}$ axial wavenumber $\left(k_{n}\right)$ and $k^{\text {th }}$ frequency $\left(\omega_{k}\right), x_{j}=j \Delta x$ is the axial coordinate of the $j^{\text {th }}$ cell, and the quantity $\phi$ is defined as

$$
\phi=k_{n} \Delta x
$$

The highest axial wavenumber that can be resolved on the mesh is $\pi / \Delta x$ by the Whittaker-Kotel'nikov-Shannon sampling theorem. This implies that $\phi$ can take on all values between $-\pi$ and $\pi$. By now inserting Eq. 24) into Eq. (23), the following is obtained

$$
\frac{\partial \hat{\hat{w}}_{k, n}^{p}}{\partial \tau}+i \omega_{k} \hat{\hat{w}}_{k, n}^{p}+\frac{\lambda_{p}}{\Delta x} \xi(\phi) \hat{\hat{w}}_{k, n}^{p}=0
$$

In this relation, $\xi(\phi)$ represents the eigenvalues of the spatial operator $F$. An analytical expression for these eigenvalues may be obtained by applying $F$ to the Fourier mode in Eq. 24). Without loss of generality, one may assume that $\lambda_{p}>0$. In this case, $\xi(\phi)$ becomes as follows

$$
\xi(\phi)=-a_{1} e^{-i 2 \phi}+\left(a_{1}-a_{2}\right) e^{-i \phi}+\left(a_{2}-a_{3}\right)+\left(a_{3}-a_{4}\right) e^{i \phi}+a_{4} e^{i 2 \phi}
$$

The coefficients $a_{1}-a_{4}$ in this equation have previously been defined in Table 1.

For the purpose of the stability analysis, Eq. 26 is now rewritten as follows 


$$
\frac{\partial \hat{\hat{w}}_{k, n}^{p}}{\partial \tau}=\underbrace{-\frac{\lambda_{p}}{\Delta x}\left(i \bar{\omega}_{k, p}+\xi(\phi)\right)}_{\Omega} \hat{\hat{w}}_{k, n}^{p}
$$

Here, $\Omega$ is used to denote the eigenvalues of the spatial + temporal discretization, and

$$
\bar{\omega}_{k, p}=\frac{\omega_{k} \Delta x}{\lambda_{p}}
$$

is the grid reduced frequency introduced by Gentilli [16]. The linear assumption introduced in Eq. (14) implies that pseudo-time integration of the original governing equations will be stable if the pseudo-time integration of Eq. (28) is stable for all values of $\Omega$. A necessary condition for this to hold is that the contour of $\Omega \Delta \tau$, where $\Delta \tau$ is the pseudo-time step, lies within the stability boundary of the pseudo-time integration scheme for all values of $\phi, \bar{\omega}_{k, p}$ and $\lambda_{p}$ [27]. The stability boundary of the pseudo-time integration scheme is further defined as the contour in the complex plane where the norm of its eigenvalue is unity [27]. Before this stability condition is analyzed, however, the product of $\Omega \Delta \tau$ is first expressed as

$$
\begin{aligned}
\Omega \Delta \tau & =-\frac{\lambda_{p} \Delta \tau}{\Delta x}\left(i \bar{\omega}_{k, p}+\xi(\phi)\right) \\
& =-\sigma\left(i \bar{\omega}_{k, p}+\xi(\phi)\right)
\end{aligned}
$$

In this equation, the well known Courant-Friedrichs-Lewy (CFL) number $\sigma$ was introduced. This number can be seen to scale the eigenvalues of the spatial+temporal discretization.

From Eq. (30) it can be noted that the eigenvalues of the temporal discretization are purely imaginary. The presence of the temporal discretization will thus move the eigenvalue spectra along the imaginary axis. If an explicit pseudo-time integration scheme is used, this can cause instabilities due to the limited stability region of these integration schemes. In order to illustrate this more clearly, contours of $\Omega \Delta \tau$ have been plotted in Fig. 1 1 together with stability boundaries of two different Runge-Kutta schemes. The contours of $\Omega \Delta \tau$ shown in this figure were obtained by varying $\phi$ in Eq. (30) between $-\pi$ and $\pi$ for different combinations of the CFL number, grid-reduced frequency, and spatial discretization scheme. The eigenvalue contours denoted TOU and TOU-LD in this figure correspond to the two, third order accurate upwind schemes defined in Table 1 In Fig. 1a, the grid reduced frequency is zero and the CFL number is $\sigma=1.2$. For this case, the stability condition can be seen to be satisfied for all combinations of pseudo-time integration and spatial discretization scheme. As shown in Fig. 1b, this is no longer the case when the CFL number is kept constant and the grid reduced frequency is increased to $\bar{\omega}_{k, p}=1$. It is thus not possible to use the same settings in both a steady state and a HB computation.

Based on the previous findings, one can think of two ways to restore stability when the HB equations are solved: select a pseudo-time integration scheme with a wider stability margin, or scale the residual using a preconditioner. A 
simple way to achieve the first option could be to switch from, e.g., a three stage to a four stage Runge-Kutta scheme when the HB equations are being solved. This would allow a similar CFL number as was used in the steady state simulation to be used for the HB simulation. A simple scaling of the residual could on the other hand be achieved by lowering the CFL number. Although this latter approach would suffer from a worse convergence rate, the total CPU time to reach convergence may be the same as the previous approach since a Runge-Kutta scheme with more stages is computationally more expensive per iteration. Given that these two approaches both have strengths and weaknesses, it is likely that the optimal setup is a combination of a tailored Runge-Kutta scheme and a good preconditioner. In the upcoming sections, one tailored Runge-Kutta scheme and two preconditioners that have been proposed in the literature will be investigated. Based on the findings obtained from this analysis, a new preconditioner for the time domain HB method will then be presented. Alternative tailored pseudo-time integration schemes will on the other hand not be considered in this work.

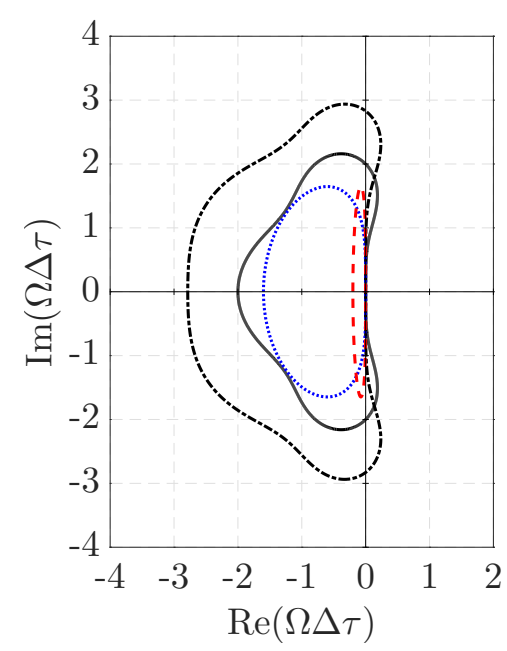

(a) $\sigma=1.2, \bar{\omega}_{k, p}=0$.

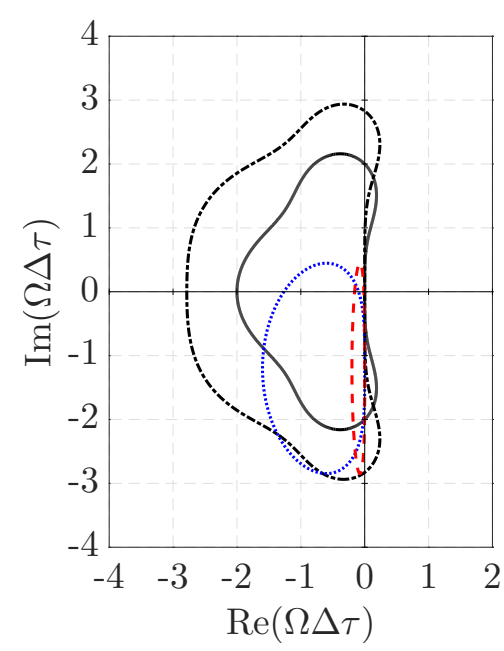

(b) $\sigma=1.2, \bar{\omega}_{k, p}=1$.

Fig. 1 Stability boundaries for two different Runge-Kutta schemes ( $-=$ RK-3, - - - - - = RK-4) together with eigenvalue spectra for different combinations of spatial discretization scheme $\left(\cdots \cdots \cdots \cdot=\mathrm{TOU},---_{-}=\right.$ TOU-LD) and grid reduced frequency.

\section{Point Implicit Runge-Kutta}

The point implicit Runge-Kutta method was originally developed by Melson et al. [28] for the dual time stepping method. Later, the application of this method to solve the HB equations was considered by McMullen [5] and Campobasso and Baba-Ahmadi [9]. The point implicit Runge-Kutta method is based on treating the term associated with the time derivative implicitly within each Runge-Kutta stage. In order to illustrate this, a general $S$ stage Runge-Kutta algorithm will first be considered. It may be written as follows [27] 


$$
\begin{aligned}
q^{(1)} & =q^{m} \\
q^{(2)} & =q^{m}+\Delta \tau \alpha_{2,1} \mathcal{R} q^{(1)} \\
& \vdots \\
q^{(s)} & =q^{m}+\Delta \tau \sum_{r=1}^{S-1} \alpha_{s, r} \mathcal{R} q^{(r)} \\
& \vdots \\
q^{(S)} & =q^{m}+\Delta \tau \sum_{r=1}^{S-1} \alpha_{S, r} \mathcal{R} q^{(r)} \\
q^{(S+1)} & =q^{m}+\Delta \tau \sum_{r=1}^{S} \beta_{r} \mathcal{R} q^{(r)} \\
q^{m+1} & =q^{(S+1)}
\end{aligned}
$$

In this algorithm, $q$ is the solution, $\mathcal{R}$ is the residual operator, $m$ is the pseudo-time time step index, and $\alpha_{s, r}, \beta_{r}$ are the Runge-Kutta coefficients. These coefficients are normally summarized in a Butcher tableau as shown in Table 2 Note that this table does not include any coefficients in the left column. The reason for this is that these coefficients are only needed if the residual explicitly depends on $\tau$, which is not the case here.

$$
\begin{array}{c|ccc}
\alpha_{2,1} & & & \\
\vdots & \ddots & & \\
\alpha_{S, 1} & \ldots & \alpha_{S, S-1} & \\
\hline \beta_{1} & \ldots & \beta_{S-1} & \beta_{S}
\end{array}
$$

\section{Table 2 Butcher tableau for Runge-Kutta coefficients.}

The point implicit Runge-Kutta method is derived from Eq. (31) by first splitting the residual operator in two parts, one representing the spatial discretization and one representing the temporal discretization. After this, the residual term corresponding to the temporal discretization is treated implicitly within each Runge-Kutta stage. This is achieved by evaluating the temporal residual as $\mathcal{R}_{\text {time }} q^{(r+1)}$, rather than as $\mathcal{R}_{\text {time }} q^{(r)}$, when the summation is performed in each Runge-Kutta stage. Due to the construction of the Runge-Kutta algorithm, all terms in this summation can be directly evaluated except the last one, which instead is moved to the left hand side. When these steps are followed, the following general form of the point implicit Runge-Kutta method is obtained 


$$
\begin{aligned}
q^{(1)} & =q^{m} \\
\left(I-\Delta \tau \alpha_{2,1} \mathcal{R}_{\mathrm{time}}\right) q^{(2)} & =q^{m}+\Delta \tau \alpha_{2,1} \mathcal{R}_{\mathrm{space}} q^{(1)} \\
& \vdots \\
\left(I-\Delta \tau \alpha_{S, S-1} \mathcal{R}_{\mathrm{time}}\right) q^{(s)} & =q^{m}+\Delta \tau \sum_{r=1}^{S-1} \alpha_{S, r} \mathcal{R}_{\text {space }} q^{(r)}+\Delta \tau \sum_{r=1}^{s-2} \alpha_{s, r} \mathcal{R}_{\mathrm{time}} q^{(r+1)} \\
& \vdots \\
\left(I-\Delta \tau \alpha_{S, S-1} \mathcal{R}_{\mathrm{time}}\right) q^{(S)} & =q^{m}+\Delta \tau \sum_{r=1}^{S-1} \alpha_{S, r} \mathcal{R}_{\mathrm{space}} q^{(r)}+\Delta \tau \sum_{r=1}^{S-2} \alpha_{S, r} \mathcal{R}_{\mathrm{time}} q^{(r+1)} \\
\left(I-\Delta \tau \beta_{S} \mathcal{R}_{\mathrm{time}}\right) q^{(S+1)} & =q^{m}+\Delta \tau \sum_{r=1}^{S} \beta_{r} \mathcal{R}_{\mathrm{space}} q^{(r)}+\Delta \tau \sum_{r=1}^{S-1} \beta_{r} \mathcal{R}_{\mathrm{time}} q^{(r+1)} \\
q^{m+1} & =q^{(S+1)}
\end{aligned}
$$

It should be noted that the operators on the left hand side in the above relation needs to be inverted before the solution can be updated in the corresponding Runge-Kutta stage. When the point implicit Runge-Kutta method is applied to Eq. 287, this amounts to a simple division since the residual operator in this equation is represented by a scalar. More precisely, the residual operator in Eq. 28] is defined as

$$
\Delta \tau \mathcal{R}=\underbrace{-i \sigma \bar{\omega}_{k, p}}_{\Delta \tau \mathcal{R}_{\text {time }}} \underbrace{-\sigma \xi(\phi)}_{\Delta \tau \mathcal{R}_{\text {space }}}
$$

In order to assess the applicability of the point-implicit Runge-Kutta method for solving the HB form of the governing equations, the stability boundary of this algorithm was calculated for two sets of Runge-Kutta coefficients and three different grid reduced frequencies using the residual operator defined in the above equation. The Runge-Kutta coefficients that were employed for this analysis are summarized in Table 3, and the resulting stability boundaries are shown in Fig. 2 The eigenvalue spectra for the two spatial discretization schemes described earlier have also been included in this figure. The reason for not including the contribution of the temporal discretization in these eigenvalue spectra is that this contribution now is taken care of by the point implicit Runge-Kutta method. From Fig. 2. it can be seen that the stability boundary for the point implicit Runge-Kutta method is rotated and expanded compared to that of the original Runge-Kutta algorithm. The expansion of the stability boundary is clearly beneficial from a stability point of view. Unfortunately, the corresponding rotation of the boundary has the opposite effect since it can uncover some eigenvalues of the spatial discretization. Figure 2 shows that this primarily happens for small values of the grid reduced frequency. This drawback of the point implicit Runge-Kutta method in the context of the HB method was originally discovered by McMullen [5]. The fact that the point implicit Runge-Kutta method can have a stabilizing 
effect for cases with high frequencies, as shown in Fig. 2c, was further reported by Campobasso and Baba-Ahmadi [9]. The stability of the point implicit Runge-Kutta method is thus dependent on the grid reduced frequency, as well as the spatial discretization scheme used. Given that the grid reduced frequency can vary a lot in the computational domain due to mesh size variations, it must however be concluded that this method can not be used as a generic approach for stabilizing explicit pseudo-time integration of the HB equations.

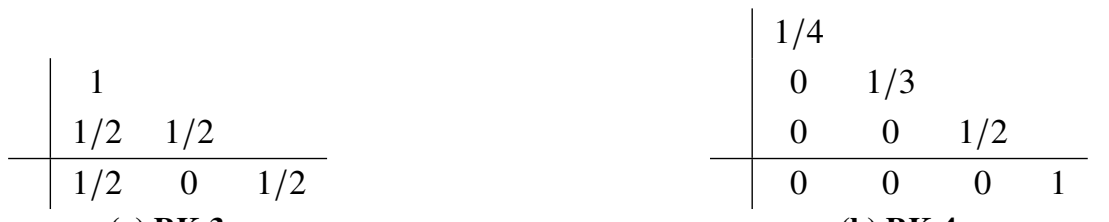

(a) RK-3 (b) RK-4

Table 3 Butcher tableau for a three stage and a four stage Runge-Kutta method.

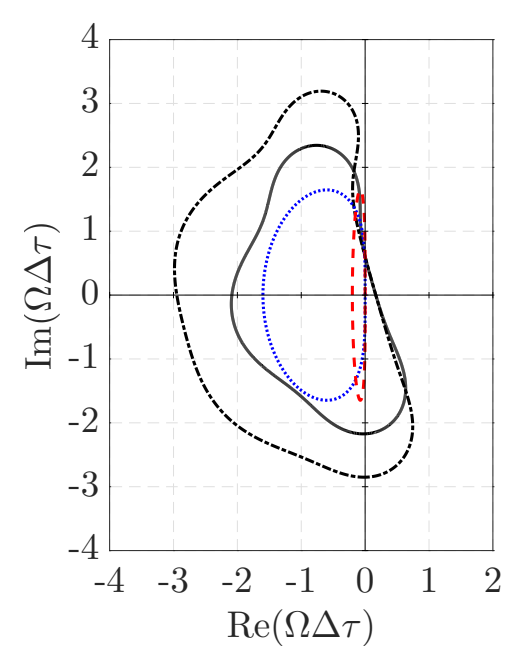

(a) $\sigma=1.2, \bar{\omega}_{k, p}=1 / 2$.

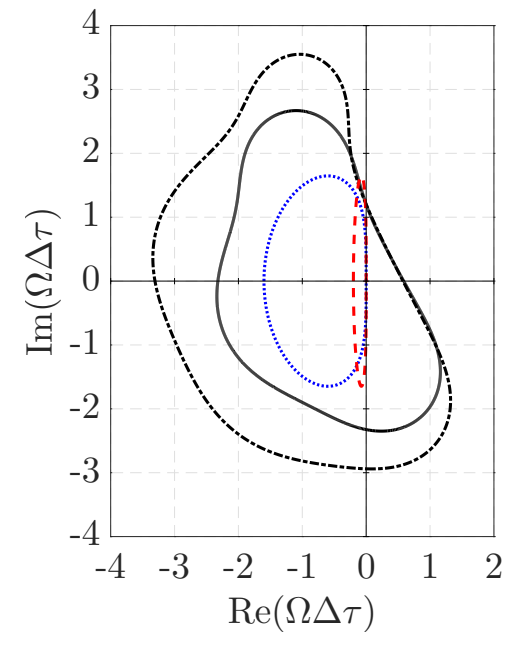

(b) $\sigma=1.2, \bar{\omega}_{k, p}=1$.

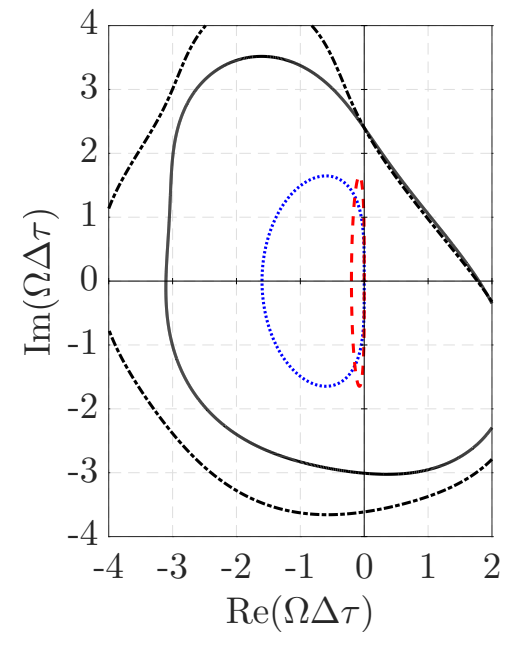

(c) $\sigma=1.2, \bar{\omega}_{k, p}=2$.

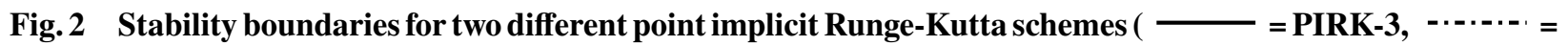
PIRK-4) together with eigenvalue spectra for two different spatial discretization schemes $(\cdots \cdots \cdots \cdot \cdot$ TOU, $-\cdot--$ = TOU-LD).

\section{HB Source Term Stabilization Matrix}

The next stabilization method that has been considered is the HB source term stabilization matrix used by Thomas et al. [17] in their two step approximate factorization approach. In this method, the time domain HB residual is multiplied by the matrix $(I+\Delta \tau \mathbf{D})^{-1}$. If this technique is applied to explicit pseudo-time integration of Eq. (28), it yields the following scaled residual

$$
\Delta \tau \mathcal{R}_{\mathrm{stab}}=\frac{1}{1+i \sigma \bar{\omega}_{k, p}}\left(-\sigma\left(i \bar{\omega}_{k, p}+\xi(\phi)\right)\right)
$$


The eigenvalue spectra defined by this residual operator is shown for three different values of the grid reduced frequency and the two spatial discretization schemes presented earlier in Fig. 3. In this figure, the stability boundaries of the two Runge-Kutta schemes presented in Table 3 have also been included. From Fig. 3 , it can be seen that the stabilization matrix scales and rotates the eigenvalue spectra. The scaling of the eigenvalue spectra is clearly an advantage from a stability point of view. The corresponding rotation can however be seen to cause stability issues for small values of the grid reduced frequency. This implies that, although the HB source term stabilization matrix ensures that the implicit pseudo-time integration used in the two step approximate factorization approach remains stable [17], this matrix unfortunately does not stabilize the explicit pseudo-time integration approach used in this work. It should however be noted that the HB source term stabilization matrix can stabilize solvers using, e.g., a first order upwind spatial discretization together with an Euler explicit pseudo-time integration scheme [20].

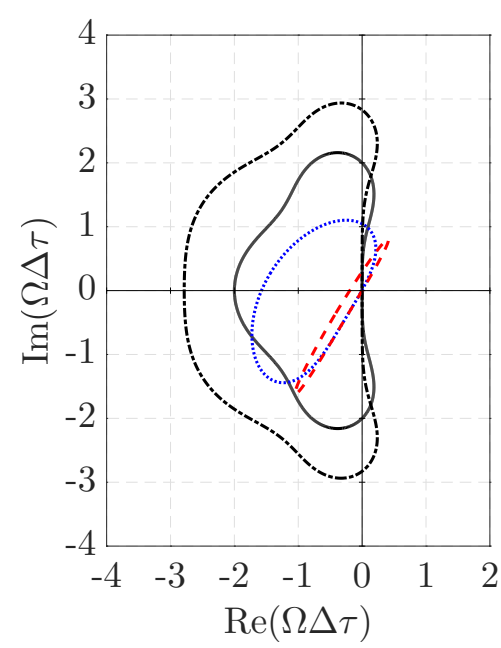

(a) $\sigma=1.2, \bar{\omega}_{k, p}=1 / 2$.

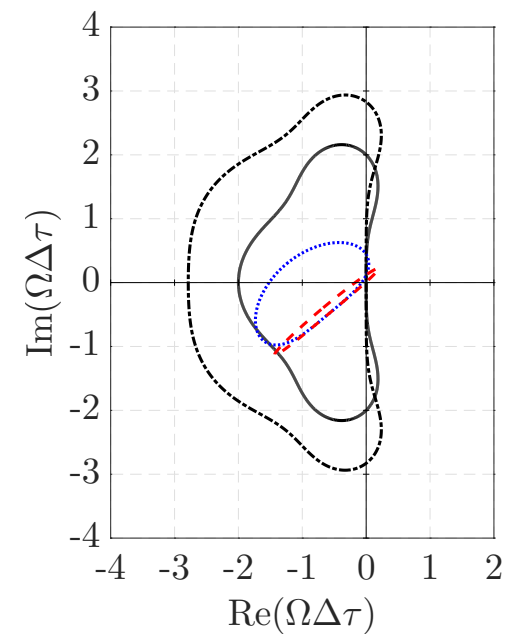

(b) $\sigma=1.2, \bar{\omega}_{k, p}=1$.

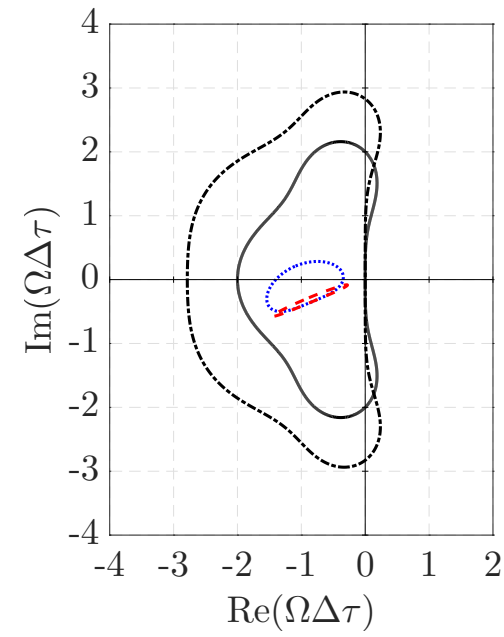

(c) $\sigma=1.2, \bar{\omega}_{k, p}=2$.

Fig. 3 Stability boundaries for two different Runge-Kutta schemes ( $-=$ RK-3, - - - - .- = RK-4) together with eigenvalue spectra obtained with the $\mathrm{HB}$ source term stabilization matrix for different combinations of spatial discretization scheme $(\cdots \cdots \cdots \cdot=$ TOU, $-\cdots---$ TOU-LD $)$ and grid reduced frequency.

\section{Restriction of Pseudo-Time Step}

Several authors have proposed to restrict the pseudo-time step in order to stabilize explicit integration of the HB equations [5, 13, 16]. Among these, the pseudo-time step restriction proposed by van der Weide et al. [13] appears to be the most widely adopted one for the time domain HB method. When this approach is applied to Eq. 28, , the residual reads as follows

$$
\Delta \tau \mathcal{R}_{\text {restrict }}=\frac{1}{1+\bar{\omega}_{K, p}}\left(-\sigma\left(i \bar{\omega}_{k, p}+\xi(\phi)\right)\right)
$$


In this equation, the residual can be seen to be scaled by a term that includes the grid reduced frequency for the largest harmonic present in the HB computation $(K)$. The reason for choosing this scaling factor is that it ensures that the corresponding pseudo-time integration of the governing equations in the time domain, where all frequencies are updated simultaneously with the same time step, remains stable [13]. The eigenvalue spectra defined by the residual operator in Eq. (35) is shown for three different values of the grid reduced frequency and the two spatial discretization schemes presented earlier in Fig. 4 In this figure, the stability boundaries of the two Runge-Kutta schemes presented in Table 3 have also been included. Figure 4 shows that the scaled pseudo-time step proposed by van der Weide et al. [13] successfully stabilizes explicit pseudo-time integration of the HB equations for all values of the grid reduced frequency. From Fig. 4a, it can also be seen that the pseudo-time step restriction is rather conservative for smaller values of the grid-reduced frequency. This is because the residual in Eq. 235) is scaled with a factor that only depends on the largest grid reduced frequency $\left(\bar{\omega}_{K, p}=2\right.$ in Fig. 47. If on the other hand Eq. (35) is adapted so that the scaling factor depends on $\left|\bar{\omega}_{k, p}\right|$, the eigenvalue spectra shown in Fig. 5 are obtained instead. From this figure, it can be seen that the adaptive scaling factor ensures that the eigenvalue spectra for the lower frequencies are scaled less than when a global scaling factor is employed. This is positive from a computational point of view since a larger scaling factor implies faster convergence. However, as noted by van der Weide et al. [13], an adaptive time step for each frequency translates into a matrix multiplication of the residual in the time domain HB method. van der Weide et al. [13] avoid this matrix multiplication by choosing the most restrictive pseudo-time step that corresponds to the highest frequency in the problem for the time domain HB method. In this work, the matrix multiplication (preconditioning) approach will instead be followed. In addition to this, the scaling factor that is applied to each frequency will be tailored based on the chosen combination of spatial discretization and pseudo-time integration scheme, similar to what has previously been done by Gentilli [16]. The derivation of this tailored preconditioner for the time domain HB method will be outlined next.

\section{Derivation of time-level Preconditioner}

The derivation of the time-level preconditioner requires that the largest pseudo-time step that can be used to update the equations in the frequency+conserved variable domain is known. The stability analysis presented in the previous section was however performed in the frequency+characteristic variable domain. In order to account for this, the pseudo-time step used to update the $k^{\text {th }}$ frequency component of the conserved variables should be selected as

$$
\Delta \tau_{k}=\min _{p}\left(\Delta \tau_{k, p}\right)
$$

Here, $\Delta \tau_{k, p}$ denotes the largest pseudo-time step that gives a stable pseudo-time integration for the $k^{\text {th }}$ frequency component of the $p^{\text {th }}$ characteristic variable. It may be calculated as 


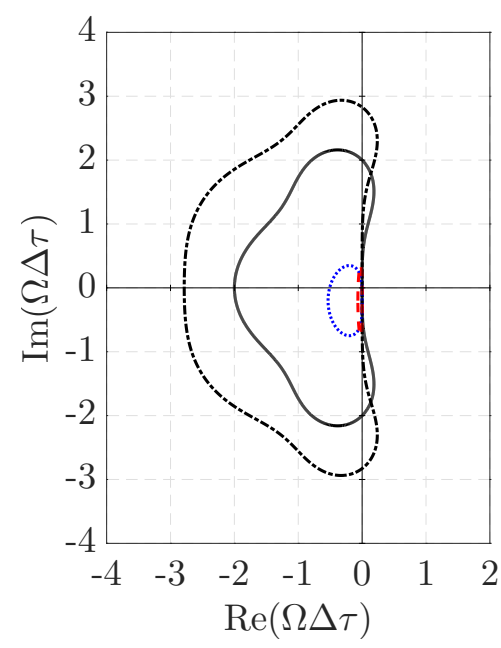

(a) $\sigma=1.2, \bar{\omega}_{k, p}=1 / 2$.

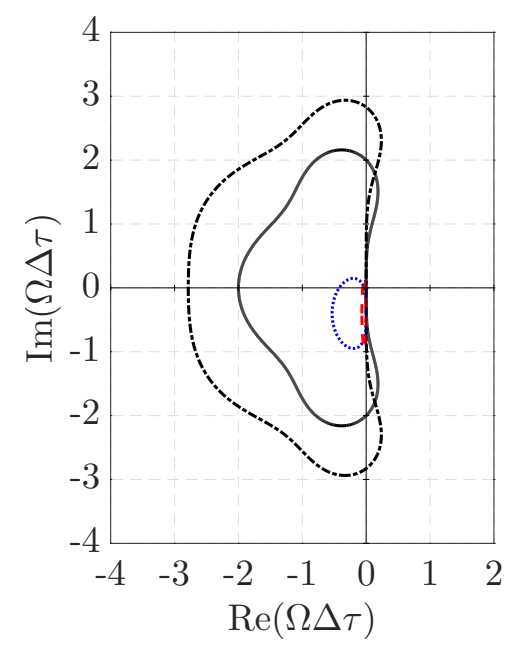

(b) $\sigma=1.2, \bar{\omega}_{k, p}=1$.

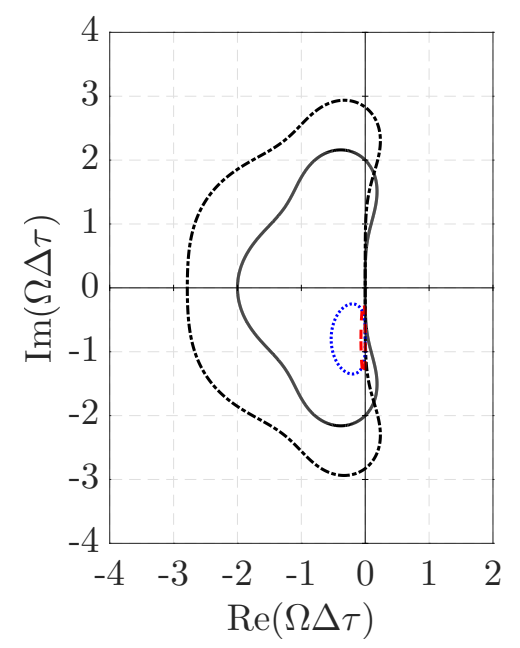

(c) $\sigma=1.2, \bar{\omega}_{k, p}=2$.

Fig. 4 Stability boundaries for two different Runge-Kutta schemes ( $=$ RK-3, -.....- = RK-4) together with eigenvalue spectra obtained with the pseudo-time step restriction defined in Eq. 35 for different combinations of spatial discretization scheme $\left(\cdots \cdots \cdots=\right.$ TOU, $-\cdots=-=^{-}=$TOU-LD) and grid reduced frequency.

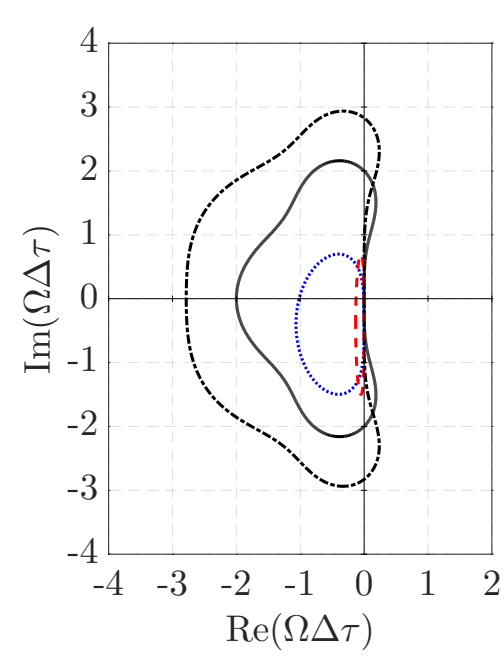

(a) $\sigma=1.2, \bar{\omega}_{k, p}=1 / 2$.

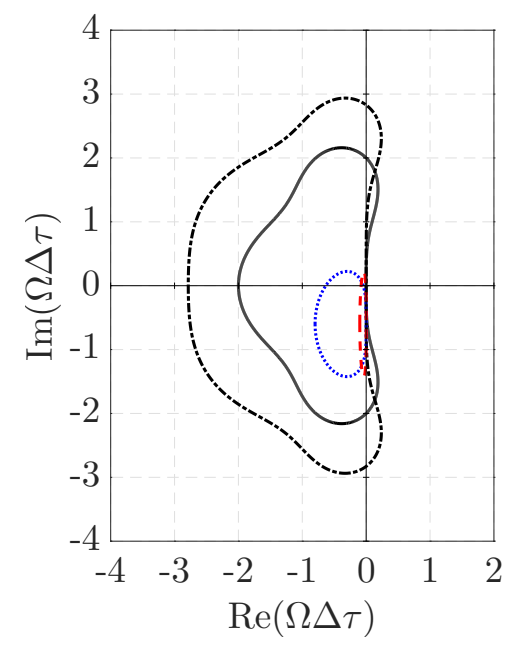

(b) $\sigma=1.2, \bar{\omega}_{k, p}=1$.

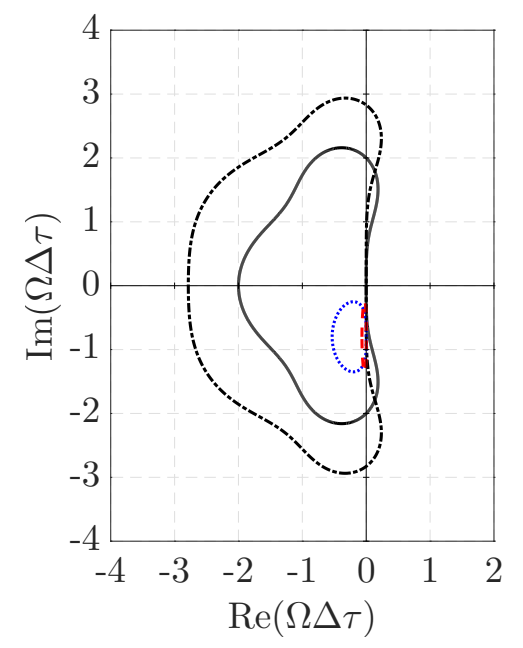

(c) $\sigma=1.2, \bar{\omega}_{k, p}=2$.

Fig. 5 Stability boundaries for two different Runge-Kutta schemes ( $-=$ RK-3, $\cdot \cdot \cdot \cdot \cdot \cdot-\mathbf{R K}-4)$ together with eigenvalue spectra obtained with an adapted scaling of the residual for different combinations of spatial discretization scheme $\left(\cdots \cdots \cdots=\right.$ TOU, $-\cdots--_{-}=$TOU-LD) and grid reduced frequency. 


$$
\Delta \tau_{k, p}=\sigma_{\mathrm{HB}}\left(\bar{\omega}_{k, p}\right) \frac{\Delta x}{\left|\lambda_{p}\right|}
$$

Here, $\sigma_{\mathrm{HB}}\left(\bar{\omega}_{k, p}\right)$ should be selected to ensure that the eigenvalue spectra defined in Eq. 30. ends up inside the stability boundary of the chosen pseudo-time integration scheme for all values of $\phi \in(-\pi, \pi)$, and the given value of $\bar{\omega}_{k, p}$. The largest value of $\sigma_{\mathrm{HB}}$ that satisfies this criteria for each grid reduced frequency has been determined for all combinations of numerical scheme and pseudo-time integration scheme considered in this work, and is shown in Fig. 6 From this figure, it can be seen that the largest allowable CFL number that ensures stability decreases as the grid reduced frequency increases. As noted previously, this follows from the fact that a non-zero grid-reduced frequency moves the eigenvalue spectra along the imaginary axis, which in turn requires the CFL number to be reduced in order for the spectra to remain inside the stability boundary.

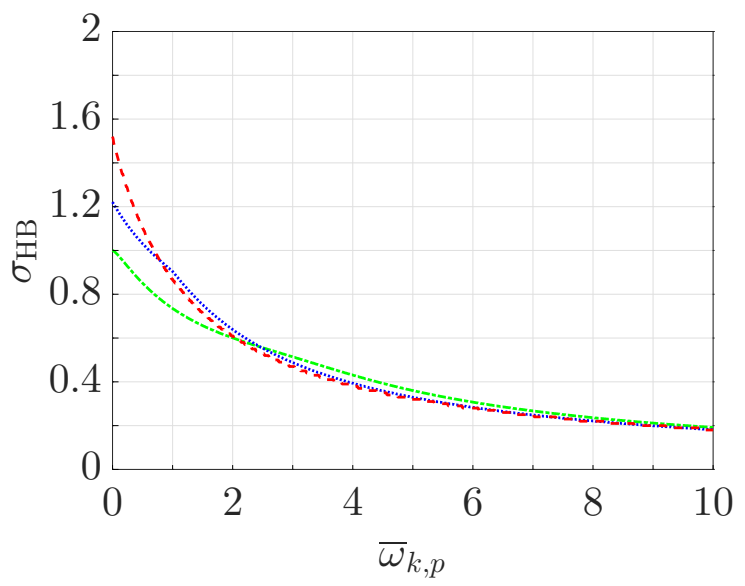

(a) RK-3.

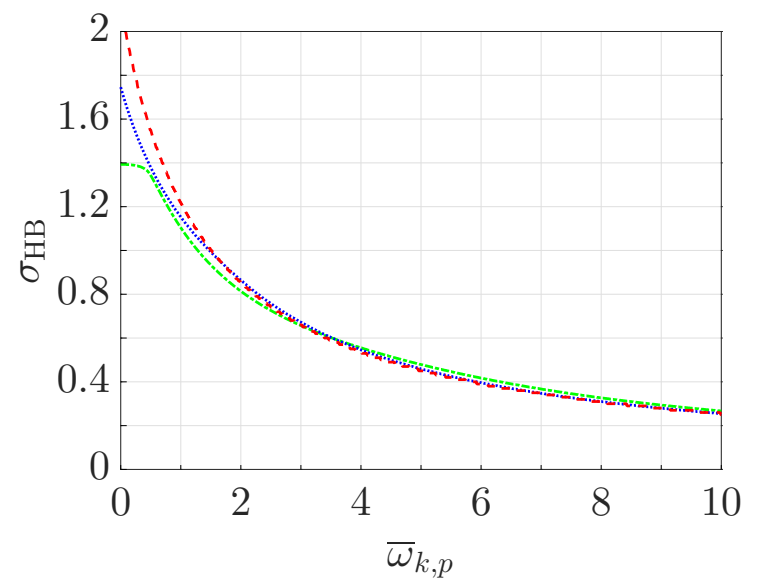

(b) RK-4.

Fig. 6 Variation of largest allowable CFL number that ensures stability with grid-reduced frequency for different combinations of spatial discretization $\left(-\cdots+=\right.$ FOU, $\cdots \cdots \cdots=$ TOU, $---{ }^{-}=$TOU-LD) and pseudo-time integration schemes.

If the CFL number shown in Fig. 6is divided by the largest CFL number that ensures stability for a steady state computation $\left(\sigma_{\text {Steady }}=\sigma_{\mathrm{HB}}(0)\right)$, the following is obtained

$$
g\left(\bar{\omega}_{k, p}\right)=\frac{\sigma_{\mathrm{HB}}}{\sigma_{\text {Steady }}}
$$

In Fig. 7 the scaling function defined by the above relation is plotted for the same combinations of spatial discretization and pseudo-time integration schemes as was shown in Fig. 6 . An approximate scaling function defined as

$$
g_{\mathrm{a}}\left(\bar{\omega}_{k, p}\right)=\frac{1}{1+\gamma\left|\bar{\omega}_{k, p}\right|}, \quad \gamma=0.75
$$

has also been included in Fig. 7. As can be seen from this figure, the approximate scaling function defined by the above 


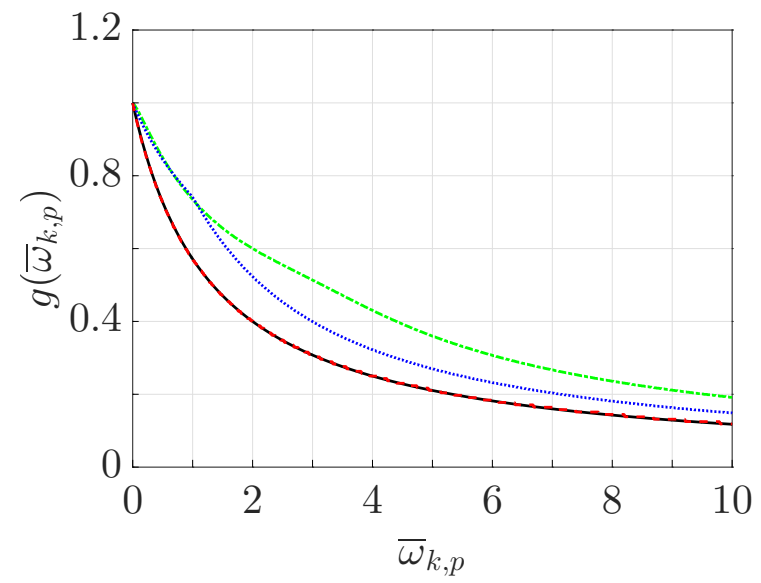

(a) RK-3.

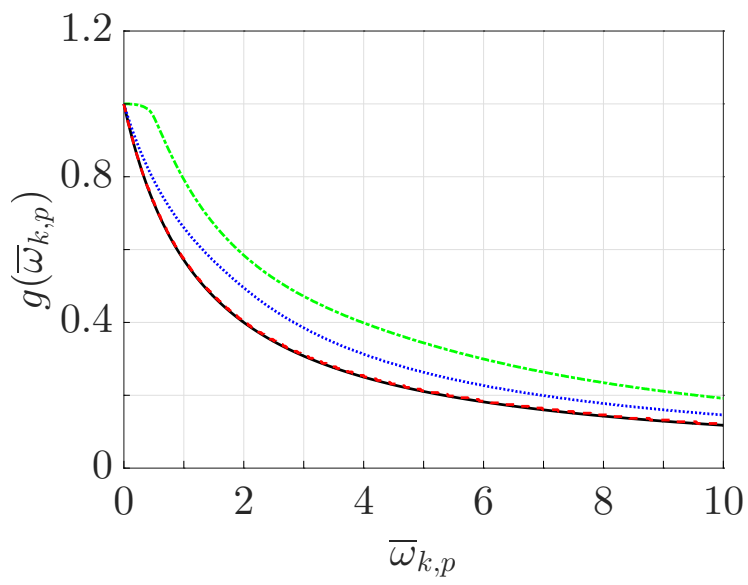

(b) RK-4.

Fig. 7 Plots of scaling function $g\left(\bar{\omega}_{k, p}\right)$ that ensures stability for different combinations of spatial discretization $(-\cdots=$ FOU, $\cdots \cdots \cdots=$ TOU, $-\cdots=$ TOU-LD) and pseudo-time integration schemes.

equation satisfies $g_{\mathrm{a}}\left(\bar{\omega}_{k, p}\right) \leq g\left(\bar{\omega}_{k, p}\right)$ for all combinations of spatial discretization, pseudo-time integration scheme and grid-reduced frequency. From this, it follows that a conservative estimate of the pseudo-time step in Eq. (37) may be obtained as

$$
\Delta \tau_{k, p}=\sigma_{\text {Steady }} g_{\mathrm{a}}\left(\bar{\omega}_{k, p}\right) \frac{\Delta x}{\left|\lambda_{p}\right|}
$$

This relation may now be combined with Eq. (36) to obtain

$$
\Delta \tau_{k}=\sigma_{\text {Steady }} \min _{p}\left(\frac{\Delta x}{\left|\lambda_{p}\right|+\gamma\left|\omega_{k}\right| \Delta x}\right), \quad \gamma=0.75
$$

From this equation, it follows that the pseudo-time step should be chosen based on the largest wave speed as

$$
\Delta \tau_{k}=\sigma_{\text {Steady }} \frac{\Delta x}{\max _{p}\left(\left|\lambda_{p}\right|\right)+\gamma\left|\omega_{k}\right| \Delta x}, \quad \gamma=0.75
$$

This expression may now be compared to the time step that would be used in a steady state computation

$$
\Delta \tau_{\text {Steady }}=\sigma_{\text {Steady }} \frac{\Delta x}{\max _{p}\left(\left|\lambda_{p}\right|\right)}
$$

The ratio between the time step that should be used in the HB computation, and the one that should be used in a steady state computation, therefore reads as follows

$$
\frac{\Delta \tau_{k}}{\Delta \tau_{\text {Steady }}}=\frac{\sigma_{\text {Steady }}}{\sigma_{\text {Steady }}+\gamma\left|\omega_{k}\right| \Delta \tau_{\text {Steady }}}, \quad \gamma=0.75
$$


Note that it is the actual frequency, and not the grid reduced frequency, that is used in the above equation.

The implementation of the preconditioner that ensures a stable pseudo-time integration may now be done as follows. First, the complete residual, including both the spatial and temporal discretization, is computed for each conserved variable in each computational cell. Next, the residual is multiplied with the same pseudo-time step that would normally be used in a steady state computation. For the one dimensional case considered here, this time step can be obtained from Eq. 43. When three dimensional, viscous flows, on the other hand are considered, the equation that relates the CFL number to the pseudo-time step will however be more complicated. Before the next Runge-Kutta stage, the residual is finally preconditioned as follows

$$
\Delta \tau \mathcal{R}_{\mathrm{prec}}^{*}=\underbrace{\mathbf{E}_{K}^{-1}(\mathbf{t}) \mathbf{G}_{K} \mathbf{E}_{K}(\mathbf{t})}_{\mathbf{P}}\left(\Delta \tau_{\text {Steady }} \mathcal{R}^{*}\right)
$$

Here, $\mathcal{R}^{*}$ denotes the residual resulting from the spatial+temporal discretization, $\mathbf{G}_{K}=G_{K} \otimes \mathbf{I}$, and

$$
G_{K}=\left[\begin{array}{cccccc}
1 & 0 & \ldots & & 0 \\
0 & \tilde{g}_{\mathrm{a}}\left(\omega_{1}\right) & 0 & \ldots & & 0 \\
\vdots & 0 & \tilde{g}_{\mathrm{a}}\left(\omega_{2}\right) & 0 & \ldots & 0 \\
& \vdots & 0 & \ddots & & 0 \\
& & \vdots & & & 0 \\
0 & 0 & 0 & 0 & 0 & \tilde{g}_{\mathrm{a}}\left(\omega_{-1}\right)
\end{array}\right]
$$

The function $\tilde{g}_{\mathrm{a}}\left(\omega_{k}\right)$ in the above equation further denotes the ratio between the stable Harmonic Balance time step and the stable steady state time step, i.e., the right hand side of Eq. 44. Note that the preconditioning matrix $\mathbf{P}$ in Eq. 45. consists of three steps. In the first step, the time domain residual of the HB equations is Fourier transformed to obtain the residual in the frequency domain. Next, each frequency component of the residual is scaled using the right hand side of Eq. (44). Finally, the scaled residual in the frequency domain is converted back to the time domain using an inverse discrete Fourier transform.

The preconditioning matrix in Eq. 45 can be written as $\mathbf{P}=P \otimes \mathbf{I}$, where $P=E_{K}^{-1}(\mathbf{t}) G_{K} E_{K}(\mathbf{t})$. For the single frequency case considered here, the elements in $P$ may be calculated analytically as

$$
P_{a, b}=\frac{1}{M}+\frac{2}{M} \sum_{k=1}^{K} \tilde{g}_{\mathrm{a}}\left(\omega_{k}\right) \cos \left(\frac{2 \pi k(a-b)}{M}\right)
$$

The preconditioning step in Eq. 45 can thus be implemented as a single vector-matrix multiplication. Note, however, that the matrix $P$ must be recalculated for each cell, since Eq. (44) depends on the local pseudo-time step. 
When the time-level preconditioner is applied to the residual in the frequency+characteristic variable domain, it reads as follows

$$
\Delta \tau \mathcal{R}_{\text {prec }}=\frac{1}{1+\gamma\left|\bar{\omega}_{k, p}\right|}\left(-\sigma\left(i \bar{\omega}_{k, p}+\xi(\phi)\right)\right), \quad \gamma=0.75
$$

The eigenvalue spectra defined by the residual operator in the above equation is shown in Fig. 8. By comparing this spectra to the one shown in Fig. 4 it can be seen that the proposed preconditioner gives a less conservative scaling of the residual than the pseudo-time step restriction proposed by van der Weide et al. [13]. The reason for this is that the proposed preconditioner scales the residual in the frequency domain, with a scaling that is tailored to the specific numerical set-up considered here.

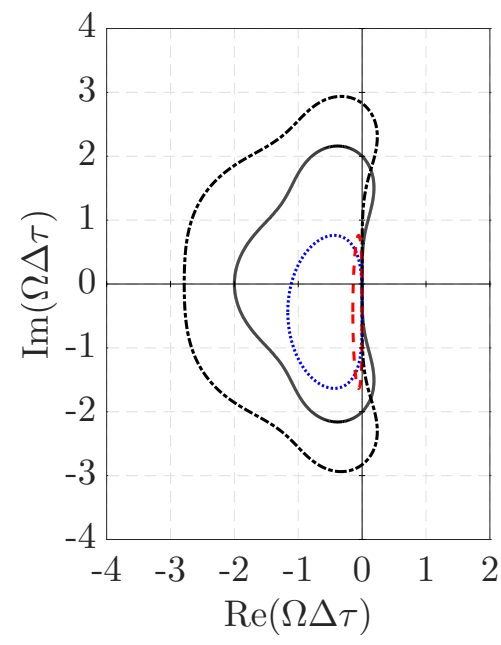

(a) $\bar{\omega}_{k, p}=1 / 2$.

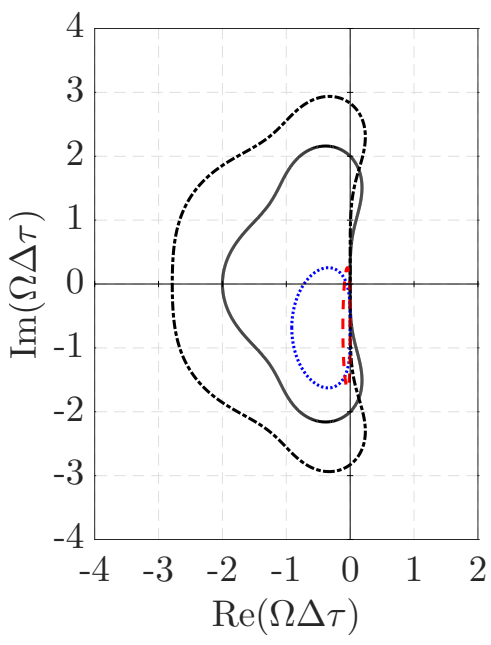

(b) $\bar{\omega}_{k, p}=1$.

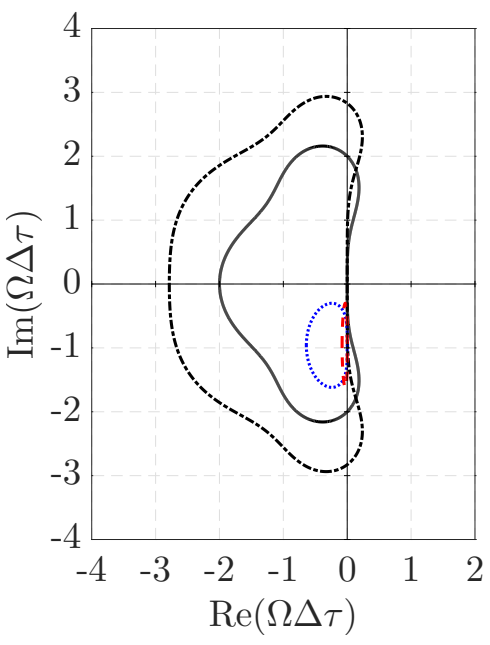

(c) $\bar{\omega}_{k, p}=2$.

Fig. 8 Stability boundaries for two different Runge-Kutta schemes ( $-=$ RK-3, - - - - - = RK-4) together with eigenvalue spectra obtained with the approximate scaling function defined in Eq. (39) for different combinations of spatial discretization scheme ( $\cdots \cdots \cdots=$ TOU, $-\cdots--_{-}=$TOU-LD) and grid reduced frequency.

The stability analysis presented in this paper was done for the linearized Euler equations in one spatial dimension. It can therefore happen that a viscous HB computation of a three dimensional problem diverges with the proposed preconditioner, despite the fact that the CFL number is set to a value which ensures that the corresponding steady state computation converges. In case this happens, the CFL number can of course be reduced slightly. In addition to reducing the CFL number, the approximate scaling function in Eq. (39) could also be modified to better suit a given numerical set up. The easiest way to do this would be to change $\gamma$. Other scaling functions, which also would be better suited to account for viscous effects, could of course also be used. Deriving such a scaling function is however out of the scope of this work. 


\section{Results}

\section{A. Inviscid flow in an Oscillating Domain}

The first test case that have been used to assess the time-level preconditioner proposed in the present work is based on computing a spatially uniform flow inside a rectangular domain that oscillates in the axial direction, see Fig. 9 The displacement of the oscillation is here defined as

$$
x(t)=A \sin \omega t
$$

Let $\bar{u}$ denote the axial component of the spatially uniform velocity field in the absolute frame of reference. From Eq. (49), it then follows that the axial velocity of the flow relative to the oscillating domain is $u(t)=\bar{u}-\omega A \cos \omega t$.

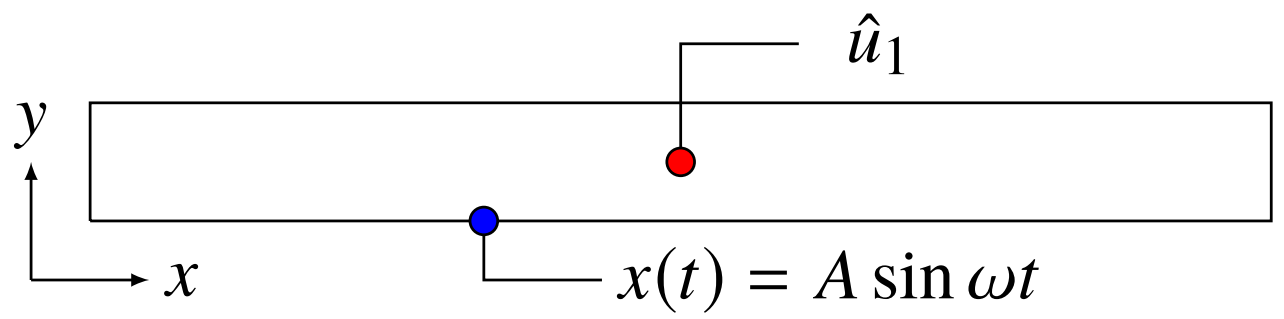

Fig. 9 Schematic view of the computational domain used in the first test case.

The flow is modeled using the two dimensional Euler equations, written in conservative form and the relative frame of reference. In order to ensure that these equations approximates the linear equations used in the stability analysis, the amplitude and frequency of the oscillation was chosen such that $\omega A=0.01 \bar{u}$. The Euler equations were solved on a uniform grid that consists of 30 cells in the axial direction, and 3 cells in the vertical direction, using the G3D::Flow code developed by the present authors. G3D::Flow uses a finite volume discretization, in which the unknown fluxes on cell faces can be reconstructed using a variety of flux schemes. In the present work, the third order upwind scheme denoted TOU-LD in Table 1 was used. At the inlet to the domain, density, velocity and pressure were specified using a non-reflecting boundary condition based on characteristic variables. These properties were chosen to give a Mach number of 0.5 with respect to the absolute frame of reference. A constant pressure and a periodic condition were further imposed at the outlet and the top/bottom boundary respectively.

The temporal derivative in the governing equations was discretized using the time domain HB method. The fundamental frequency in the HB method was set equal to the oscillation frequency of the domain, which in turn was selected such that the grid reduced frequency with respect to the largest wave speed became $\omega \Delta x /(\bar{u}+\bar{c})=2 / 3$. The largest grid reduced frequency in the problem was then adjusted by including more harmonics in the HB computation.

For each number of harmonics, three different setups were evaluated, one without any stabilization strategy, one using the pseudo-time step restriction proposed by van der Weide et al. [13], and one using the time-level preconditioner 
proposed in the present work. In all simulations, the three stage Runge-Kutta scheme defined in Table 3 a was used to integrate the equation in pseudo-time. It should also be noted that all simulations were performed with the largest CFL number that ensured convergence, and that no additional stabilization method such as implicit residual smoothing was used.

The convergence history of the first Fourier coefficient of the axial velocity component (monitored in the middle of the domain, see Fig. 97 is shown for the HB computations that contain one harmonic of the fundamental frequency in Fig. 10a. Note that this metric should satisfy $2 \operatorname{Re}\left(\hat{u}_{1}\right)=-\omega A$ when the simulation is converged. From Fig. 10a. it can be seen that all three simulations converge to this value in approximately the same number of iterations. This similar convergence rate comes from the fact that the first frequency component of the solution, which is the dominant component in this problem, is updated with approximately the same time step in all three simulations. Note, however, that this time step is computed differently in each simulation. In the simulation that does not employ any stabilization strategy, it is computed based on a reduced CFL number. In the other two simulations, the CFL number is on the other hand set to the same value that would be used in a steady state simulation, and then scaled before it is used to compute the time step in each computational cell. The similar convergence rate for all three simulations that contain one fundamental frequency is also evident from Fig. 11a, which shows the convergence history for the axial momentum residual.

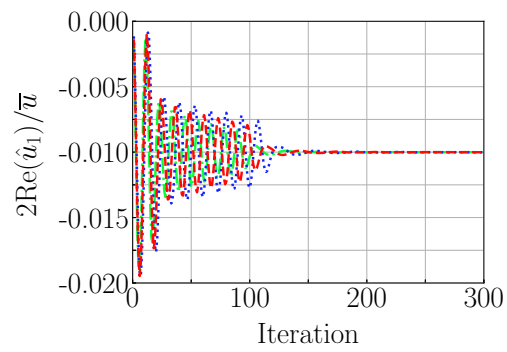

(a) HB-1.

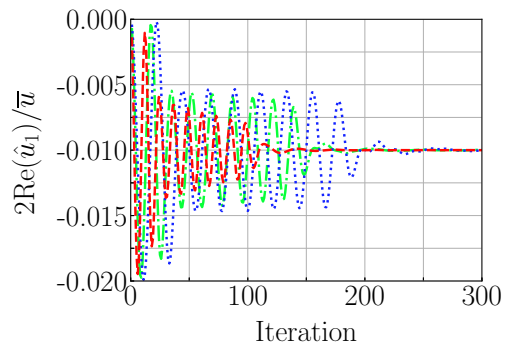

(b) HB-3.

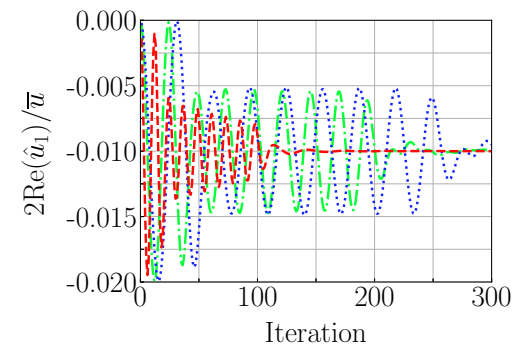

(c) HB-5.

Fig. 10 Convergence history of $\operatorname{Re}\left(\hat{u}_{1}\right)$ for different numerical setups. $\quad$ No stabilization method ( $\sigma=1.1$ for HB-1, $\sigma=0.7$ for HB-3, $\sigma=0.5$ for HB-5), $\cdots \ldots \ldots \ldots=$ pseudo-time step restriction proposed by van der Weide et al. [13], = - - = = time-level preconditioner proposed in present work.

The convergence history for $\operatorname{Re}\left(\hat{u}_{1}\right)$ is shown for all simulations that include three harmonics and five harmonics of the fundamental frequency in Fig. $10 \mathrm{~b}$ and $10 \mathrm{c}$ respectively. These figures show that the convergence rate starts to differ between the three setups when the number of harmonics increase. This is also evident from the residual plots shown in Figs $11 \mathrm{~b}$ and $11 \mathrm{c}$ The different convergence rate seen in these figures comes from the fact that the largest grid reduced frequency increases as the number of harmonics increases. From this, it follows that a simulation that employs either a reduced CFL number, or the pseudo-time step restriction, will converge slower, since these simulations scale the residual in the time domain, and thus must account for the largest grid reduced frequency in the problem. The time-level 
preconditioner proposed in the present work does on the other hand scale the residual in the frequency domain. From this, it follows that each frequency component of the solution can be updated with an individual time step. In particular, the lowest frequency component in the solution, which is the dominant component in this problem, will be updated with the same time step, independently on the number of harmonics included in the computation. This explains why the time-level preconditioner converges in the same number of iterations, independently on the number of harmonics included in the problem.

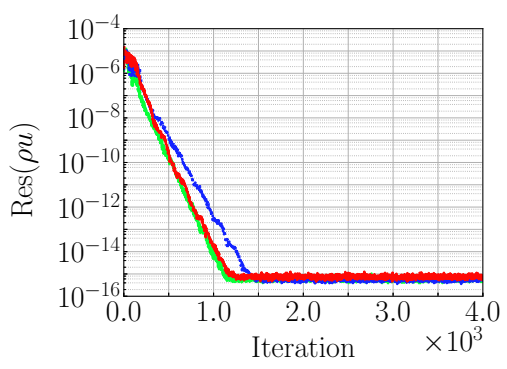

(a) HB-1.

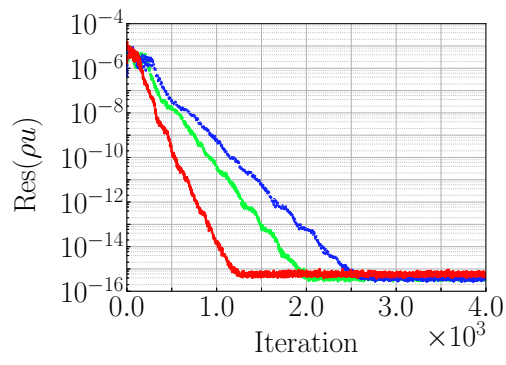

(b) HB-3.

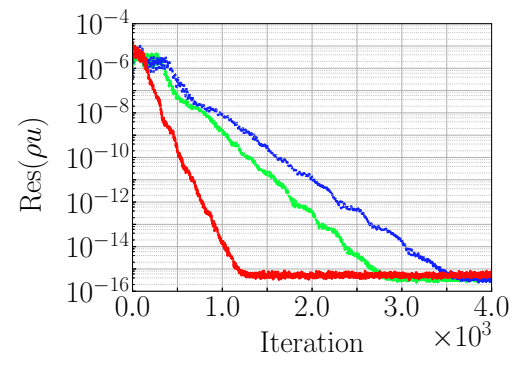

(c) HB-5.

Fig. 11 Convergence history of $\operatorname{Res}(\rho u)$ for different numerical setups. $-\cdots=$ No stabilization method ( $\sigma=1.1$ for HB-1, $\sigma=0.7$ for HB-3, $\sigma=0.5$ for HB-5), $\cdots \cdots \cdots \cdots=$ pseudo-time step restriction proposed by van der Weide et al. [13], - - - - = time-level preconditioner proposed in present work.

Although the proposed preconditioner converges in fewer iterations, the total wall-clock time of a simulation is arguably a more relevant metric in terms of performance. In order to evaluate this metric, a normalized time per iteration was computed for each simulation. It is defined as follows

$$
\text { Normalized Time Per Iteration }=\left(\frac{\text { Time Per Iteration }}{M}\right) /\left(\frac{\text { Time Per Iteration }}{M}\right)_{\text {ref }}
$$

Here, the reference simulation is taken to be the HB-1 simulation that did not employ any stabilization approach. The normalized time per iteration is shown for all simulations in Table 4. By comparing these values to the convergence plots shown in Figs 10 and 11, it is found that the time-level preconditioner also performs best in terms of total wall clock time for the first test case.

\begin{tabular}{llll}
\hline & CFL & TSR & TLP \\
HB-1 & 1.00 & 0.99 & 1.08 \\
HB-3 & 1.01 & 0.98 & 1.29 \\
HB-5 & 1.05 & 1.02 & 1.43 \\
\hline
\end{tabular}

Table 4 Normalized time per iteration (see Eq. (50p) for oscillating domain test case. CFL = Simulation employing a reduced CFL number (no stabilization method), TSR = pseudo-time step restriction proposed by van der Weide et al. [13], TLP = time-level preconditioner proposed in present work. 


\section{B. Laminar Vortex Shedding}

The second case that was used to evaluate the time-level preconditioner proposed in the present work is two dimensional, laminar vortex shedding behind a circular cylinder at $\operatorname{Re}=\frac{\rho U_{\infty} d}{\mu}=185$. The computational domain used for all simulations was constructed using a cylindrical o-grid with an outer diameter of $100 \mathrm{~d}, 280$ cells in the radial direction and 320 cells in the circumferential direction. As shown in Fig. 12, these cells were distributed to ensure that the majority of them were situated in the wake region and close to the cylinder wall. The size of the cells in the wall normal direction at the cylinder surface was further set to $0.0075 \mathrm{~d}$.

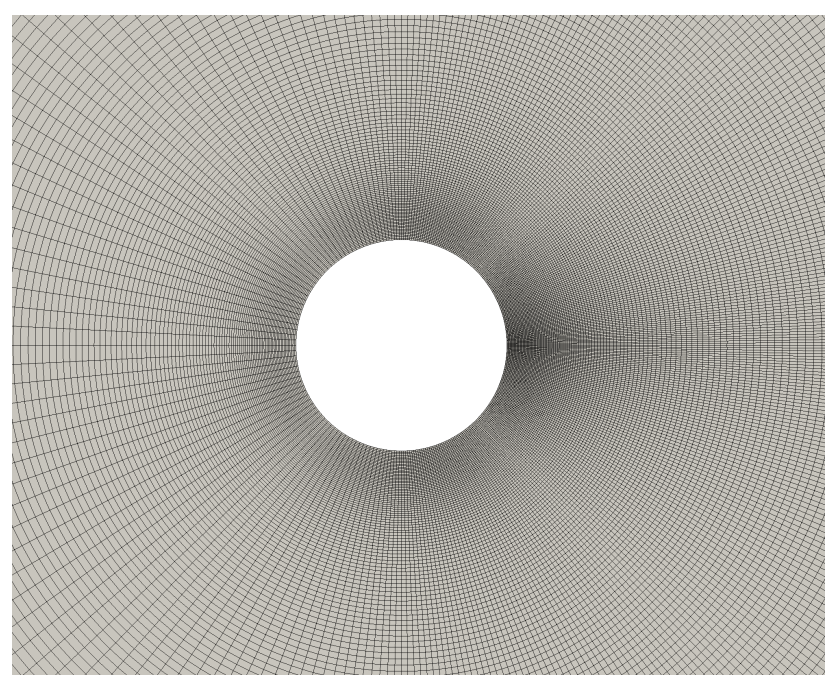

Fig. 12 Close up view of the computational mesh.

The vortex shedding simulations were performed by solving the compressible Navier-Stokes equations in conservative form using the G3D::Flow code. The convective fluxes arising from the finite volume discretization employed in G3D::Flow were once again estimated with the TOU-LD scheme, whereas the diffusive fluxes were estimated with a second order centered difference scheme. The cylinder was modeled as a no-slip wall. At the far field boundary, the same nonreflecting boundary condition as used in the previous test case was imposed. The freestream state was set to give the desired Reynolds number and a Mach number of 0.2 . This Mach number was selected to give a virtually incompressible flow, while at the same time avoiding bad convergence due to a large discrepancy between acoustic and convective wave speeds.

Before the HB simulations were run, a reference time-accurate simulation was performed with the three stage Runge-Kutta scheme presented in Table 3a This simulation was performed with a nondimensional time step of $\frac{U_{\infty} \Delta t}{d}=0.00026$ and gave a Strouhal number of $\mathrm{St}=0.192$. This Strouhal number is compared to results published by other authors in Table 5. The frequency of the vortex shedding obtained from the time-accurate simulation was used as the fundamental frequency in the HB computations. Two sets of HB computations were performed, one with $K=6$ harmonics and one with $K=8$ harmonics. For each of these sets, three different setups were used, one without 


\begin{tabular}{lccc}
\hline & St & $\bar{C}_{D}$ & $C_{L, \mathrm{rms}}$ \\
RK-3 & 0.192 & 1.356 & 0.456 \\
HB-6, $(\sigma=0.7)$ & - & 1.355 & 0.454 \\
HB-6-TSR, $(\sigma=1.4)$ & - & 1.355 & 0.454 \\
HB-6-TLP, $(\sigma=1.4)$ & - & 1.355 & 0.454 \\
HB-8, $(\sigma=0.6)$ & - & 1.356 & 0.456 \\
HB-8-TSR, $(\sigma=1.4)$ & - & 1.356 & 0.456 \\
HB-8-TLP, $(\sigma=1.4)$ & - & 1.356 & 0.456 \\
Lu and Dalton [29] & 0.195 & 1.31 & 0.422 \\
Guilmineau and Queutey [30] & 0.195 & 1.287 & 0.443 \\
\hline
\end{tabular}

Table 5 Summary of results obtained with time-accurate solver, with HB solver and by other authors. TSR = pseudo-time step restriction proposed by van der Weide et al. [13], TLP = time-level preconditioner presented in this work.

any preconditioning, one with the pseudo-time step restriction proposed by van der Weide et al. [13] and one with the time-level preconditioner presented in this work. In all simulations, the three stage Runge-Kutta scheme presented in Table 3a was used for pseudo-time integration using the largest CFL number that ensured convergence. Apart from local time stepping, no other acceleration methods such as multi-grid or implicit residual smoothing were used to ensure that the effects of the pseudo-time step restriction/time-level preconditioner could be isolated. A summary of the results obtained, including results reported by other authors, is provided in Table 5 . From this table, it can be seen that the results obtained with the G3D::Flow code agree well with previously published results. It is also interesting to note that the drag and lift coefficients obtained from the HB simulations approach those obtained with the time-accurate simulation as the number of harmonics increases. The results obtained for a fixed number of harmonics do also not depend on whether the preconditioner or pseudo-time step restriction is used, as should be expected.

The convergence history of the drag coefficient is presented for all the HB simulations in Fig. 13. In this figure, the drag coefficient obtained from the time accurate simulation has also been included for reference. From Fig. 13, it can be seen that both the pseudo-time step restriction proposed by van der Weide et al. [13] and the time-level preconditioner presented in this work give faster convergence than an HB simulation that only uses the CFL number to control the pseudo-time step.

The convergence history of the lift coefficient is presented for all the different HB simulations in Fig. 14 In this figure, the lift coefficient obtained from the time accurate simulation has also been included for reference. The trends in Fig. 14 very much resemble those shown previously for the drag coefficient. This shows that both the pseudo-time step restriction proposed by van der Weide et al. [13] and the time-level preconditioner presented in this work can give better convergence than when the CFL number simply is used to determine the local pseudo-time step.

The convergence histories of the momentum residual for all HB simulations performed are finally shown in Fig. 15 This figure shows that the convergence of the HB method greatly benefits from employing either the pseudo-time step 


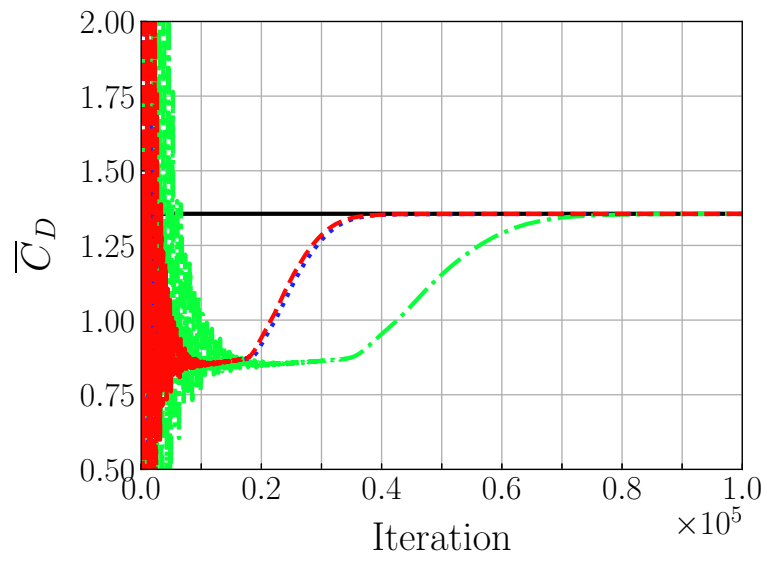

(a) HB-6.

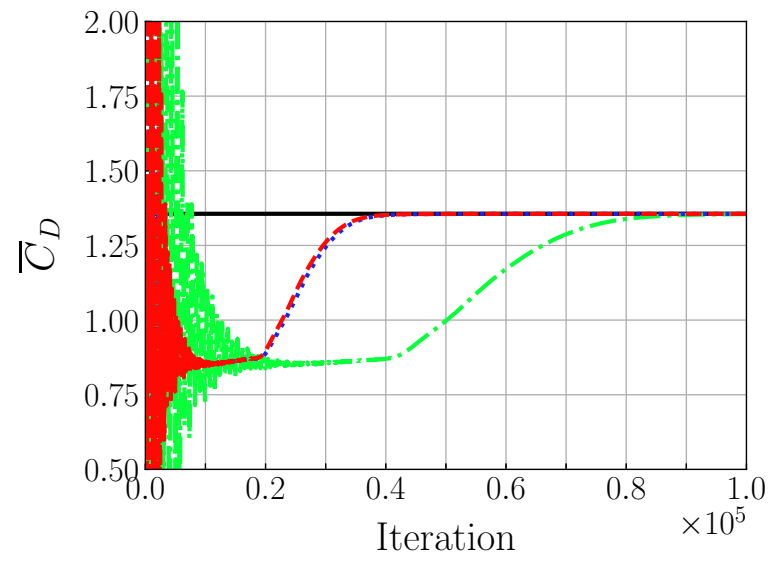

(b) HB-8.

Fig. 13 Convergence history of $\bar{C}_{D}$ for different numerical setups. $\cdots=$ No stabilization method $(\sigma=0.7$ for HB-6, $\sigma=0.6$ for HB-8), $\cdots \cdots \cdots \cdot$ = pseudo-time step restriction proposed by van der Weide et al. [13], - - - - = time-level preconditioner proposed in present work, - = reference value from time accurate simulation using the RK-3 scheme.

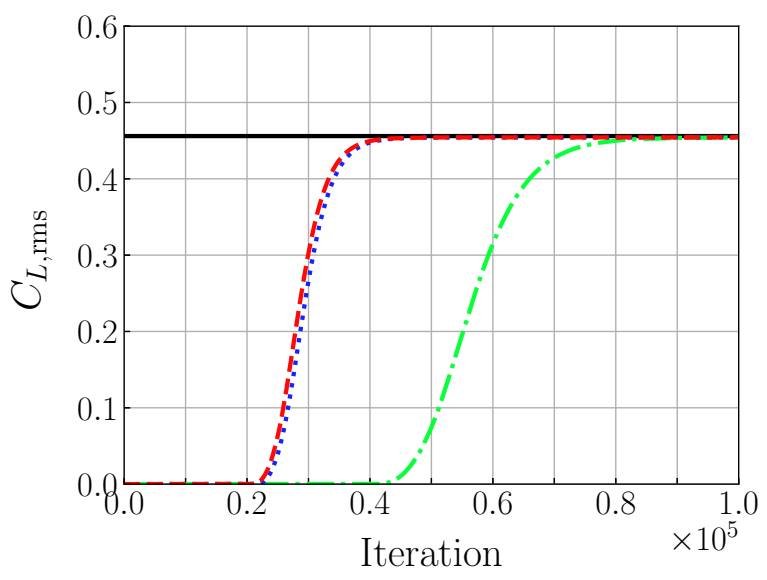

(a) HB-6.

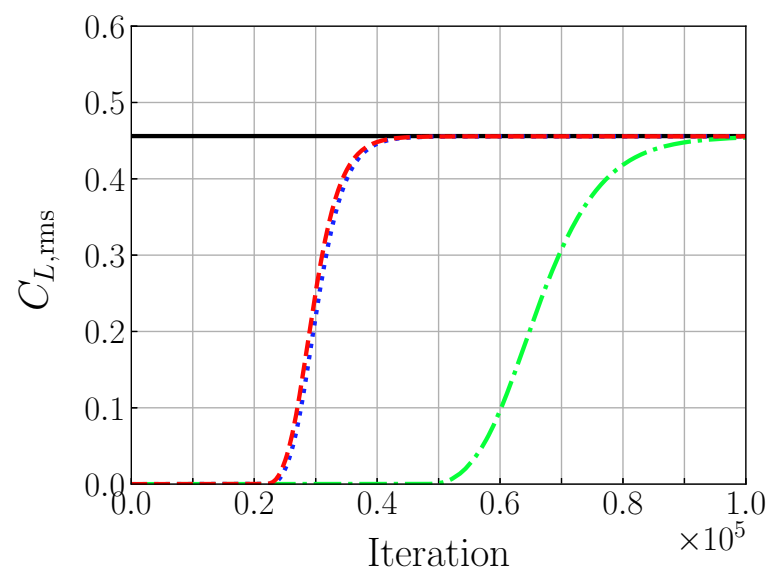

(b) HB-8.

Fig. 14 Convergence history of $C_{L, \mathrm{rms}}$ for different numerical setups. $(\sigma=0.7$ for HB-6, $\sigma=0.6$ for HB-8), $\cdots \cdots \cdots \cdot=$ pseudo-time step restriction proposed by van der Weide et al. [13], - - - - = time-level preconditioner proposed in present work, - = reference value from time accurate simulation using the RK-3 scheme. 


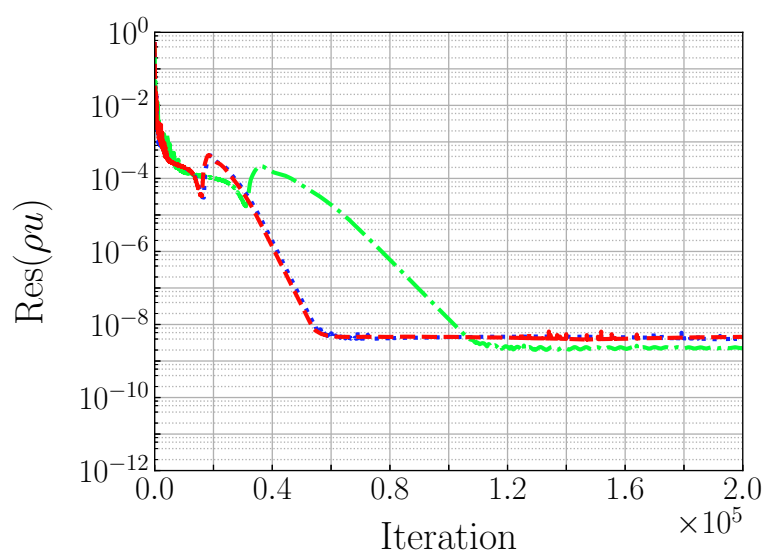

(a) HB-6.

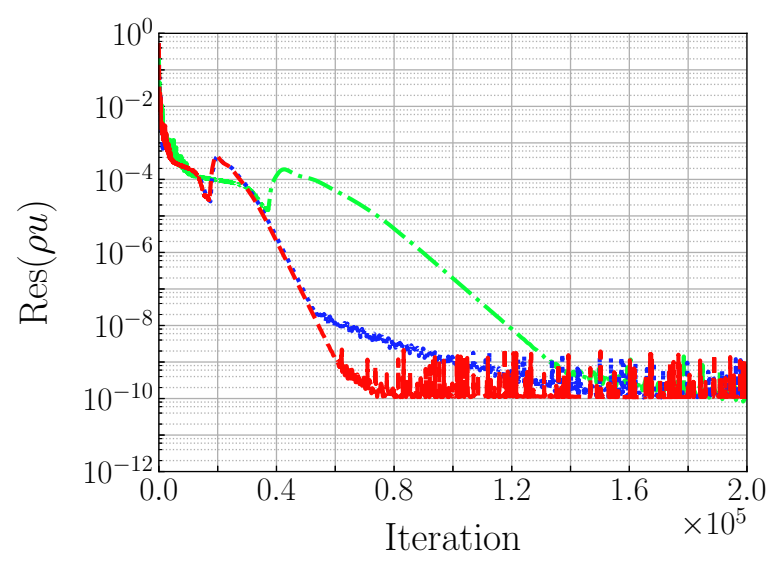

(b) HB-8.

Fig. 15 Convergence history of $\operatorname{Res}(\rho u)$ for different numerical setups. $\quad-\cdots$ ( $\sigma=0.7$ for HB-6, $\sigma=0.6$ for HB-8), $\cdots \cdots \cdots \cdot$. pseudo-time step restriction proposed by van der Weide et al. [13], - - - - = time-level preconditioner proposed in present work.

restriction or the time-level preconditioner. The oscillations in the residual seen in Fig. $15 \mathrm{~b}$ are further thought to originate from some small amplitude oscillations in pseudo-time that is not efficiently damped by the low-dissipation TOU-LD scheme.

The reason why the convergence suffers when the CFL numbers is used to determine the pseudo-time step is that it must be selected to ensure that the integration of the governing equations remains stable in all computational cells. This implies that cells in the far field, which typically are large and thus have a large grid reduced frequency, must be accounted for when the CFL number is selected. If on the other hand the pseudo-time step restriction or time-level preconditioner is employed, a local adjustment of the pseudo-time step will be done in each cell based on the grid-reduced frequency in that cell. This means that close to the cylinder, where the grid reduced frequency is significantly smaller, these two methods give a less restrictive scaling of the residual. Given that most flow variations also occur in the well resolved region close to the cylinder, it follows that the time-level preconditioner and pseudo-time step restriction should give better convergence as well.

The assumption that the grid-reduced frequency is small in the cells close to the cylinder can further be proven as follows: Assume that the flow only contains small amplitude perturbations that propagate through convection. Assume further that the mesh has been designed to give $n_{p}$ points per wavelength for the highest frequency perturbation in the flow. Given that this perturbation propagates at speed $\lambda_{p}$, it then follows that the largest grid reduced frequency becomes

$$
\frac{\omega_{K} \Delta x}{\lambda_{p}}=\frac{2 \pi}{n_{p}}
$$

This equation shows that the grid-reduced frequency will be small in well resolved regions $\left(n_{p}\right.$ large $)$.

The normalized time per iteration defined in Eq. 50, was also computed for this test case. The reference simulation 
was here selected to be the HB-6 computation that did not employ any stabilization strategy. The resulting normalized time per iteration for the second test case are shown in Table6. By comparing these results to the convergence plots considered earlier, it can be seen that the most efficient method in terms of total wall-clock time for the second test case is the pseudo-time step restriction proposed by van der Weide et al. [13], followed by the time-level preconditioner proposed in the present work.

\begin{tabular}{llll}
\hline & CFL & TSR & TLP \\
HB-6 & 1.00 & 0.96 & 1.34 \\
HB-8 & 0.98 & 0.97 & 1.56 \\
\hline
\end{tabular}

Table 6 Normalized time per iteration (see Eq. (50)) for cylinder vortex shedding test case. CFL $=$ Simulation employing a reduced CFL number (no stabilization method), TSR = pseudo-time step restriction proposed by van der Weide et al. [13], TLP = time-level preconditioner proposed in present work.

\section{Conclusion}

In this work, a thorough stability analysis of the Harmonic Balance method has been performed. This stability analysis showed that the time derivative source term employed in the Harmonic Balance method adds an imaginary part to the eigenvalue spectra of the spatial discretization, as was previously shown by McMullen [5]. The imaginary part added by the time derivative was also shown to be proportional to the grid reduced frequency introduced by Gentilli [16]. Given that explicit pseudo-time integration schemes such as, e.g., the Runge-Kutta method, only are stable when the eigenvalue spectra lies in a limited region in the complex plane, the present stability analysis helps to explain why explicit Harmonic Balance solvers can encounter stability issues when the grid reduced frequency is large, as was previously shown by Gentilli [16].

Three approaches for stabilizing the Harmonic Balance method, namely the point implicit Runge-Kutta method [9. 12], the Harmonic Balance source term stabilization matrix [31], and the pseudo-time step restriction proposed in [13], have also been investigated using stability analysis. Although these methods have been proven valuable in the particular solvers used in the cited references, only the pseudo-time step restriction proposed in [13] provided a generic stabilization approach for the numerical methods tested in this work (explicit Runge-Kutta pseudo-time integration and third order upwind finite volume schemes).

Based on knowledge gained from the stability analysis, a time-level preconditioner was also developed. This preconditioner can be seen as a tailored version of the pseudo-time step restriction proposed by van der Weide et al. [13] in the sense that the preconditioner updates each frequency component in the solution with a different pseudo-time step, and that the pseudo-time step is chosen to fit the spatial discretization scheme used. This preconditioner was then evaluated for two test cases. In the first case, a spatially uniform, inviscid flow through an oscillating domain was computed. The benefit of this test case is that it has a simple analytical solution, and that it isolates the effect of the CFL 
number and the grid reduced frequency on the stability and convergence of the solver. In particular, it was shown that the time-level preconditioner proposed in the present work gave better convergence than simulations that employed either no stabilization method, or the pseudo-time step restriction proposed in [13]. In the second case, laminar vortex shedding behind a circular cylinder was considered. For this test case, the time-level preconditioner and the pseudo-time step restriction converged twice as fast as the simulation that simply used the CFL number to calculate the pseudo-time step. The reason for this speed up is that the pseudo-time step restriction and the time-level preconditioner can use a larger local-time step in each cell by accounting for the local grid-reduced frequency. Although the time-level preconditioner has a theoretical advantage over the pseudo-time step restriction, no significant advantage was observed in the second test case. The reason for this is thought to stem from the fact that most variations in the flow occur in regions where the grid is well resolved, and consequently, the grid-reduced frequency is small. If on the other hand multi-grid and/or a less well resolved grid is employed, the time-level preconditioner is expected to have a larger advantage. Although the time-level preconditioner was developed and demonstrated for explicit pseudo-time integration schemes, it could also be used to improve convergence of implicit schemes by increasing the diagonal dominance of the linear system.

\section{Acknowledgments}

This work was financially supported by the Swedish Energy Agency under the HIGH2 2 project, Grant Agreement No 2017-00116. The authors would also like to acknowledge the Swedish National Infrastructure for Computing (SNIC) for providing computer resources at the Chalmers Centre for Computational Science and Engineering $\left(\mathrm{C}^{3} \mathrm{SE}\right)$ in Gothenburg, Sweden

\section{References}

[1] He, L., "Fourier Methods for Turbomachinery Applications," Progress in Aerospace Sciences, Vol. 46, No. 8, 2010 , pp. $329-341$. doi https://doi.org/10.1016/j.paerosci.2010.04.001

[2] Krack, M., and Groß, J., Harmonic Balance for Nonlinear Vibration Problems, Mathematical Engineering, Springer International Publishing, Gewerbestrasse 11, 6330, Cham, Switzerland, 2019. doi 10.1007/978-3-030-14023-6

[3] He, L., and Ning, W., "Efficient Approach for Analysis of Unsteady Viscous Flows in Turbomachines," AIAA Journal, Vol. 36, No. 11, 1998, pp. 2005-2012. doi $10.2514 / 2.328$

[4] Hall, K. C., Jeffrey, P. T., and Clark, W. S., "Computation of Unsteady Nonlinear Flows in Cascades Using a Harmonic Balance Technique," AIAA Journal, Vol. 40, No. 5, 2002, pp. 879-886. doi 10.2514/2.1754

[5] McMullen, M. S., “The Application of Nonlinear Frequency Domain Methods to the Euler and Navier-Stokes Equations,” Ph.D. thesis, Department of Aeronautics and Astronautics, Stanford University, Stanford, CA, 2003.

\footnotetext{
*Heat load distribution In hot parts of industrial Gas turbines burning HYDROGEN rich fuel
} 
[6] Hall, K. C., Ekici, K., Thomas, J. P., and Dowell, E. H., "Harmonic Balance Methods Applied to Computational Fluid Dynamics Problems," International Journal of Computational Fluid Dynamics, Vol. 27, No. 2, 2013 , pp. $52-67$. doi $10.1080 / 10618562.2012 .742512$.

[7] Frey, C., Kersken, H.-P., Ashcroft, G., and Voigt, C., "A Harmonic Balance Technique for Multistage Turbomachinery Applications," ASME Turbo Expo 2014: Turbine Technical Conference and Exposition, Düsseldorf, Germany, 2014. doi $10.1115 /$ GT2014-25230

[8] Sicot, F., Dufour, G., and Gourdain, N., “A Time-Domain Harmonic Balance Method for Rotor/Stator Interactions,” Journal of Turbomachinery, Vol. 134, No. 1, 2012, pp. 011001-1-011001-13. doi:10.1115/1.4003210.

[9] Campobasso, M. S., and Baba-Ahmadi, M. H., "Analysis of Unsteady Flows Past Horizontal Axis Wind Turbine Airfoils Based on Harmonic Balance Compressible Navier-Stokes Equations With Low-Speed Preconditioning,” Journal of Turbomachinery, Vol. 134, No. 6, 2012, pp. 061020-1-011001-13. doi $10.1115 / 1.4006293$.

[10] Huang, H., and Ekici, K., "An Efficient Harmonic Balance Method for Unsteady Flows in Cascades," Aerospace Science and Technology, Vol. 29, No. 1, 2024, pp. 144-154. doi https://doi.org/10.1016/j.ast.2013.02.004

[11] McMullen, M. S., Jameson, A., and Alonso, J. J., "Acceleration of Convergence to a Periodic Steady State in Turbomachinery Flows," 39th AIAA Aerospace Sciences Meeting and Exhibit, Reno, NV, 2001. doi 10.2514/6.2001-152

[12] McMullen, M. S., and Jameson, A., “The Computational Efficiency of Non-Linear Frequency Domain Methods,” Journal of Computational Physics, Vol. 212, No. 2, 2006, pp. 637-661. doi 10.1016/j.jcp.2005.07.021

[13] van der Weide, E., Gopinath, A. K., and Jameson, A., “Turbomachinery Applications with the Time Spectral Method,” 35th AIAA Fluid Dynamics Conference and Exhibit, Toronto, ON, Canada, 2005. doi $10.2514 / 6.2005-4905$

[14] Su, X., and Yuan, X., "Implicit solution of time spectral method for periodic unsteady flows," International Journal for Numerical Methods in Fluids, Vol. 63, No. 7, 2010, pp. 860-876. doi 10.1002/fld.2111.

[15] Guédeney, T., Gomar, A., Gallard, F., Sicot, F., Dufour, G., and Puigt, G., "Non-uniform time sampling for multiplefrequency harmonic balance computations," Journal of Computational Physics, Vol. 236, No. 1, 2013, pp. 317-345. doi https://doi.org/10.1016/j.jcp.2012.11.010

[16] Gentilli, N. C., "Efficient Solution of Unsteady Nonlinear Flows Using a Multiple Zone Harmonic Balance Technique," Master's thesis, Department of Mechanical Engineering and Materials Science, Duke University, Durham, NC, Dec. 2010.

[17] Thomas, J. P., Custer, C. H., Dowell, E. H., Hall, K. C., and Corre, C., "Compact Implementation Strategy for a Harmonic Balance Method Within Implicit Flow Solvers,” AIAA Journal, Vol. 51, No. 6, 2013, pp. 1374-1381. doi 10.2514/1.J051823.

[18] Woodgate, M. A., and Badcock, K. J., "Implicit Harmonic Balance Solver for Transonic Flow with Forced Motions," AIAA Journal, Vol. 47, No. 4, 2009, pp. 893-901. doi $10.2514 / 1.36311$ 
[19] Antheaume, S., and Corre, C., "Implicit Time Spectral Method for Periodic Incompressible Flows," AIAA Journal, Vol. 49, No. 4, 2011, pp. 791-805. doi $10.2514 / 1 . J 050785$

[20] Custer, C. H., “A Nonlinear Harmonic Balance Solver for an Implicit CFD Code: OVERFLOW 2," Ph.D. thesis, Department of Mechanical Engineering and Materials Science, Duke University, Durham, NC, 2009.

[21] Sicot, F., Puigt, G., and Montagnac, M., "Block-Jacobi Implicit Algorithms for the Time-Spectral Method," AIAA Journal, Vol. 46, No. 13, 2008, pp. 3080-3089. doi $10.2514 / 1.36792$

[22] Wang, D. X., and Huang, X., "Solution Stabilization and Convergence Acceleration for the Harmonic Balance Equation System," Journal of Engineering for Gas Turbines and Power, Vol. 139, No. 9, 2017, pp. 092503-2-092503-9. doi:10.1115/1.4035912

[23] Kundert, K. S., White, J. K., and Sangiovanni-Vincentelli, A., Steady-State Methods for Simulating Analog and Microwave Circuits, The Kluwer international series in engineering and computer science, Vol. 94, Kluwer Academic Publishers, 101 Philip Drive, Assinippi Park, Norwel, MA 02061 USA, 1990. doi $10.1007 / 978-1-4757-2081-5$.

[24] Junge, L., Ashcroft, G., Kersken, H.-P., and Frey, C., “On The Development of Harmonic Balance Methods for Multiple Fundamental Frequencies," ASME Turbo Expo 2018: Turbomachinery Technical Conference and Exposition, Oslo, Norway, 2018. doi $10.1115 / \mathrm{GT} 2018-75495$.

[25] Nimmagadda, S., Economon, T. D., Alonso, J. J., and da Silva, C. R. I., "Robust uniform time sampling approach for the harmonic balance method," 46th AIAA Fluid Dynamics Conference, Washington, D.C., 2016. doi 10.2514/6.2016-3966

[26] Andersson, N., "A Study of Subsonic Turbulent Jets and Their Radiated Sound Using Large-Eddy Simulation,” Ph.D. thesis, Division of Fluid Dynamics, Department of Applied Mechanics, Chalmers University of Technology, Gothenburg, Sweden, 2005.

[27] Hirsch, C., Numerical Computation of Internal and External Flows, $2^{\text {nd }}$ ed., Vol. 1, Butterworth-Heinemann, 2007. doi https://doi.org/10.1016/B978-0-7506-6594-0.X5037-1.

[28] Melson, N. D., Atkins, H. L., and Sanetrik, M. D., “Time-accurate Navier Stokes calculations with multigrid acceleration,” The 6th Copper Mountain Conference on Multigrid Methods, Copper Mountain, CO, 1993.

[29] Lu, X.-Y., and Dalton, C., "Calculation of the Timing of Vortex Formation from an Oscillating Cylinder," Journal of Fluids and Structures, Vol. 10, No. 5, 1996, pp. 527-541. doi $10.1006 /$ jfls.1996.0035.

[30] Guilmineau, E., and Queutey, P., "A Numerical Simulation of Vortex Shedding from an Oscillating Circular Cylinder," Journal of Fluids and Structures, Vol. 16, No. 6, 2002, pp. 773-794. doi 10.1006/jfls.2002.0449

[31] Thomas, J. P., Custer, C. H., Dowell, E. H., and Hall, K. C., "Unsteady Flow Computation Using a Harmonic Balance Approach Implemented about the OVERFLOW 2 Flow Solver," 19th AIAA Computational Fluid Dynamics Conference, San Antonio, TX, 2009. doi $10.2514 / 6.2009-4270$ 